\title{
NONLINEAR STATE OBSERVERS AND EXTENDED KALMAN FILTERS FOR BATTERY SYSTEMS
}

\author{
ANDREAS RAUH, SAIF S. BUTT, HARALD ASCHEMANN \\ Chair of Mechatronics \\ University of Rostock, Justus-von-Liebig-Weg 6, D-18059 Rostock, Germany \\ e-mail: \{Andreas.Rauh, Saif.Butt, Harald.Aschemann\}@uni-rostock.de
}

\begin{abstract}
The focus of this paper is to develop reliable observer and filtering techniques for finite-dimensional battery models that adequately describe the charging and discharging behaviors. For this purpose, an experimentally validated battery model taken from the literature is extended by a mathematical description that represents parameter variations caused by aging. The corresponding disturbance models account for the fact that neither the state of charge, nor the above-mentioned parameter variations are directly accessible by measurements. Moreover, this work provides a comparison of the performance of different observer and filtering techniques as well as a development of estimation procedures that guarantee a reliable detection of large parameter variations. For that reason, different charging and discharging current profiles of batteries are investigated by numerical simulations. The estimation procedures considered in this paper are, firstly, a nonlinear Luenberger-type state observer with an offline calculated gain scheduling approach, secondly, a continuous-time extended Kalman filter and, thirdly, a hybrid extended Kalman filter, where the corresponding filter gains are computed online.
\end{abstract}

Keywords: observers, state estimation, Riccati equations, extended Kalman filters, parameter estimation.

\section{Introduction}

Battery systems are integral components in hybrid electric power train structures. Recent advances in both efficiency and energy density of battery systems allow their usage as a secondary power source in various applications. These applications range from hybrid drive chains in automotive applications to load shaping devices for fuel cell systems. Moreover, batteries can be used as intermediate storage elements to provide an additional degree of freedom for the decoupling of the operating point in an internal combustion engine from the drive cycle. In this case, batteries are charged both during regenerative braking phases and by means of a load level increase of the internal combustion engine. The current research does not only focus on automotive or truck applications but also on the hybridization of even larger diesel engines. In the work of Leska et al. (2011), for example, batteries are used as a secondary power source for hybrid railway vehicles.

To fully exploit the recuperation capabilities of a battery by means of reliable control and/or state estimation procedures, it is necessary to develop a reasonably low-dimensional mathematical model that adequately describes the dynamic behavior of the battery.
The complexity of mathematical battery models ranges from static and quasi-static descriptions to complex electro-chemical models (Benger et al., 2009; Gomadam et al., 2002). In the case of static and quasi-static modeling, the relationship between battery parameters, operating conditions as well as terminal voltages and currents is either described by a set of algebraic equations or by numeric data represented in lookup tables (Johnson, 2002). For this reason, static and quasi-static models require only small computational effort. However, these models are insufficient to account for the dynamics during the charging and discharging of batteries. Hence, they are not suitable to implement accurate control and estimation strategies for dynamic operating conditions. Moreover, these static descriptions do not allow detection of aging phenomena such as degradation of the battery performance, which is related to an increasing internal resistance or to a decreasing internal battery capacitance. Aging occurs due to various factors such as temperature variations, capacity loss due to long-lasting time of storage, and repeated charging and discharging cycles.

To overcome the lack of information with respect to the battery dynamics and their corresponding aging effects, which goes along with using (quasi-) static 
battery models, the development of more detailed electro-chemical descriptions has been considered, for example, by Gu and Wang (2000), Wang and Srinivasan (2002), Klein et al. (2012) or Smith et al. (2010). Such electro-chemical models are based on mathematical formulations of the chemical reactions taking place inside batteries.

Commonly, these models are represented by a set of partial differential equations with a large number of parameters that are not directly accessible by measurements in commercial applications. To allow real-time implementations of control and state estimation procedures for batteries, a trade-off between static and quasi-static descriptions on the one hand and high-dimensional electro-chemical models on the other is inevitable. Such system models, in the following referred to as control-oriented models, are typically based on electric equivalent circuits that can be described by sets of ordinary differential equations or differential algebraic equations (Salameh et al., 1992; Buller et al., 2005; Benini et al., 2001; Aylor et al., 1992). Control-oriented models allow introducing model simplifications-such as constant operating temperatures-to adapt the degrees of freedom with respect to the application at hand. Furthermore, as shown in this paper, aging phenomena can be easily included by expressing them as variations of the battery parameters. As an alternative to the presented observer approaches, fault estimation techniques such as those described, for example, by Xu et al. (2012) could be applied to detect aging-relevant parameters.

In any application of batteries in both purely electric and hybrid drive systems, knowledge of the amount of energy available from the battery is essential for the reliability of control and operating strategies. This amount of energy imposes a restriction on how long a battery can be used without requiring further recharging. Mathematically, this energy can be given as a function of the state of charge. The higher the state of charge, the longer the battery can provide energy, and vice-versa.

In addition, accurate knowledge of the state of charge is a crucial prerequisite to keep its value within those limits that guarantee maximum battery life time. Since the only measured data available from the battery in practical applications are the charging and discharging currents as well as the terminal voltage, it is not possible to determine the state of charge directly. This leads to the need for state estimation techniques that adapt automatically to a varying state of health of the battery. Various techniques to estimate the state of charge have been proposed in previous works (cf. Aylor et al., 1992; Zhang et al., 2008; Bhangu et al., 2005; Kim, 2006; Chiasson and Vairamohan, 2005).

Zhang et al. (2008) estimated the state of charge by using a linear relationship between the open circuit voltage and the state of charge itself. This estimation approach, hence, is only applicable for quasi-static operating conditions in a restricted range for the state of charge. Neural network methods for state of charge estimation such as those proposed by Chan et al. (2000), Bo et al. (2008) or Shen (2010) do not require linearity assumptions between the open circuit voltage and the state of charge. However, this method suffers from problems due to the computational burden for the training of the neural network involving a large number of data points. Representatives for state of charge estimation procedures relying on dynamic system models given by sets of ordinary differential equations are, for example, sliding mode observers as described by Zhang et al. (2008) or Kim (2006). The effects of uncertain parameters as well as battery aging have not been examined explicitly in the above-mentioned estimation techniques.

To handle such phenomena, a nonlinear Luenberger-type state observer has been proposed by Rauh et al. (2010). The results of this paper are briefly summarized in Section 3.1. The Luenberger-type observer is designed in such a way as to estimate parameter variations as well as the state variables of equivalent circuit models for batteries under both nominal and pre-aged operating conditions. This observer technique is compared with a Continuous-Time implementation of an Extended Kalman Filter (CT-EKF) as well as with a Hybrid Extended Kalman Filter (H-EKF). The underlying battery model, taken from the works of Chen and Rincon-Mora (2006) as well as Erdinc et al. (2009), is based on an experimental identification of the state of charge dependencies of all system parameters. In such a way, practical applicability can be guaranteed for all estimation procedures presented in this paper.

The structure of this paper is as follows. In Section 2 a control-oriented mathematical model of the battery is stated that takes into account the explicit relationship between the parameters of an equivalent circuit model and the state of charge of the battery. This mathematical model is extended to include parameter variations resulting from degradation. In Section 3, different nonlinear model-based observer and filtering techniques exploiting either an offline or an online computation of the respective gains are described in detail along with their mathematical formulations. Corresponding simulation results are provided in Section 4 for both nominal operating conditions and disturbed parameters. These simulations are carried out to compare the performance of the Luenberger-type state observer, the CT-EKF and the $\mathrm{H}-\mathrm{EKF}$, with the aim to identify operating conditions for which either of the approaches is more reliable. Finally, conclusions and an outlook on future work are given in Section 5 . 


\section{Control-oriented mathematical models for battery systems}

As mentioned before, purely static and quasi-static battery models cannot be employed to quantify the dynamic behavior during charging and discharging phases, whereas electro-chemical models often involve a huge number of internal parameters that cannot be identified properly in real-time applications. Therefore, the control-oriented modeling approach is preferred to develop different estimation procedures in the following. Such control-oriented models range from purely ohmic networks to RC network representations taking into account charging and discharging effects. Pang et al. (2001) proposed a simplified purely resistive network as an equivalent circuit model for batteries. Since batteries are typically exposed to time-varying charging and discharging currents, for example, in power train applications, the Thevenin equivalent circuit including RC networks to account for charging and discharging cycles has been proposed by Salameh et al. (1992), Buller et al. (2005), Benini et al. (2001) or Aylor et al. (1992). In the Thevenin equivalent circuit model, lumped RC networks are introduced which allow studying the effects of both slow and fast charging cycles. Further extensions of these battery models, directly incorporating aging effects, can be found, e.g., in the works of Serrao et al. (2005), Rong and Pedram (2006) or Remmlinger et al. (2011).

In this paper, an extended battery model based on Thevenin's equivalent circuit model is used. The basic battery model is shown in Fig. 1, where the shaded blocks represent aging by parameter variations as described in Section 2.2. The electric equivalent circuit contains two first-order lag elements to describe the dynamics of charging and discharging with different time constants.

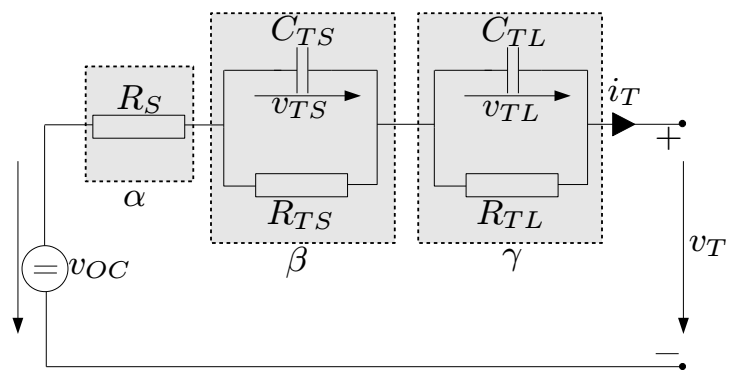

Fig. 1. Extended equivalent circuit of a battery.

As a model simplification, temperature variations are neglected in this contribution. The effect of temperature variations has been described by Rauh and Aschemann (2012) by introducing it as an additional state variable in the dynamic battery model. The following derivation of a battery model is divided into two parts: first, a nominal model of the battery is developed, where all equivalent circuit parameters are assumed to be accurately known; second, parameter variations and uncertainties are introduced in the mathematical model to deal with aging effects resulting in the extended battery model presented by Rauh et al. (2010), as well as Rauh and Aschemann (2012).

2.1. Nominal battery model. In the nominal battery model, the state of charge $\sigma(t)$ as well as the voltages $v_{T L}(t)$ and $v_{T S}(t)$ across the RC networks shown in Fig. 1 represent the state variables, whereas the terminal current $i_{T}(t)$ is the known input applied to the battery. The terminal voltage $v_{T}(t)$ is employed as the measured system output.

The state of charge is considered to be a normalized quantity $\sigma(t) \in[0 ; 1]$, where $\sigma(t)=1$ corresponds to the fully charged battery and $\sigma(t)=0$ represents the completely discharged battery. Assuming exactly known values for the terminal current $i_{T}(t)$ and the battery capacitance $C_{\text {Bat }}$, the state of charge can be determined by integrating the first-order differential equation

$$
\dot{\sigma}(t)=-\frac{i_{T}(t)}{C_{\mathrm{Bat}}}
$$

Unfortunately, the battery capacitance $C_{\mathrm{Bat}}$ depends on external effects such as aging and temperature variations and is therefore not precisely known. For this reason, it is essential to consider measured data along with (1) to reconstruct $\sigma(t)$ (for which the initial condition $\sigma(0)$ cannot be measured directly) over sufficiently long time intervals. In the nominal model, the open circuit voltage $v_{O C}(t)$ is a function of the state of charge $\sigma(t)$ (Chen and Rincon-Mora, 2006; Erdinc et al., 2009; Rauh et al., 2010), leading to the necessity to include it explicitly as a state variable. The battery model summarized in the remainder of Section 2.1 is based on the works of Chen and Rincon-Mora (2006) as well as Erdinc et al. (2009), where both the structure of the equivalent circuit shown in Fig. 1 and its parameterizations have been validated experimentally for nominal operating conditions of a new battery that has not yet been exposed to aging.

The relationship between the open circuit voltage $v_{O C}(t)$ and the state of charge $\sigma(t)$ can be described by a parameterizable nonlinear algebraic function

$$
\begin{aligned}
v_{O C}(\sigma(t))= & v_{0} \cdot e^{v_{1} \cdot \sigma(t)}+v_{2}+v_{3} \cdot \sigma(t) \\
& +v_{4} \cdot \sigma^{2}(t)+v_{5} \cdot \sigma^{3}(t) .
\end{aligned}
$$

The corresponding nonlinear characteristic for the open circuit voltage is displayed in Fig. 2

Ohmic losses are taken into account by a series connection of the internal resistance of the battery, denoted by $R_{S}(t)$, with two RC subnetworks. This resistance,

$$
R_{S}(t)=R_{S a} \cdot e^{R_{S b} \cdot \sigma(t)}+R_{S c}
$$




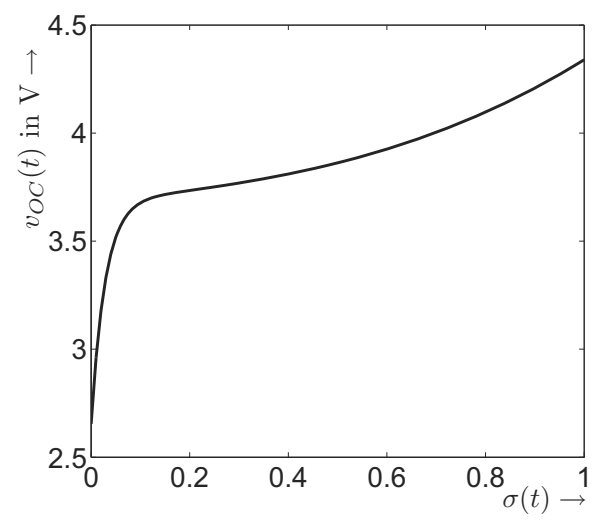

Fig. 2. Open circuit voltage $v_{O C}(t)=v_{O C}(\sigma(t))$ as a function of the state of charge $\sigma(t)$.

depends on the state of charge $\sigma(t)$ in a nonlinear way.

As shown in Fig. 11 the charging and discharging dynamics are modeled by the previously mentioned RC subnetworks accounting for processes with short and large time constants ( $T S$ and $T L$, respectively). The parameter values of the RC subnetworks can be identified experimentally on the basis of an impedance spectroscopy. They typically show an exponential dependency on the state of charge $\sigma(t)$, where the parameters are given by

$$
\begin{aligned}
& R_{T S}(t)=R_{T S a} \cdot e^{R_{T S b} \cdot \sigma(t)}+R_{T S c}, \\
& C_{T S}(t)=C_{T S a} \cdot e^{C_{T S b} \cdot \sigma(t)}+C_{T S c}, \\
& R_{T L}(t)=R_{T L a} \cdot e^{R_{T L b} \cdot \sigma(t)}+R_{T L c}, \\
& C_{T L}(t)=C_{T L a} \cdot e^{C_{T L b} \cdot \sigma(t)}+C_{T L c},
\end{aligned}
$$

according to Chen and Rincon-Mora (2006), as well as Erdinc et al. (2009). The voltages of the RC subnetworks are represented by $v_{T S}(t)$ and $v_{T L}(t)$ according to the differential equations

$$
\dot{v}_{T S}(t)=\frac{-v_{T S}(t)}{C_{T S}(t) \cdot R_{T S}(t)}+\frac{i_{T}(t)}{C_{T S}(t)}
$$

and

$$
\dot{v}_{T L}(t)=\frac{-v_{T L}(t)}{C_{T L}(t) \cdot R_{T L}(t)}+\frac{i_{T}(t)}{C_{T L}(t)} .
$$

Finally, the terminal voltage $v_{T}(t)$ can be computed by an evaluation of Kirchhoff's voltage law as the algebraic equation

$$
v_{T}(t)=v_{O C}(t)-i_{T}(t) \cdot R_{S}(t)-v_{T S}(t)-v_{T L}(t) .
$$

It can be seen from Eqns. (10-10 that all parameters in the equivalent circuit model are in general nonlinear functions of the state of charge $\sigma(t)$.
For further investigations, the mathematical model of the nominal system is written in the compact form

$$
\dot{\mathbf{x}}(t)=\mathbf{f}(\mathbf{x}(t), u(t)),
$$

with the state vector

$$
\mathbf{x}(t):=\left[\begin{array}{lll}
\sigma(t) & v_{T S}(t) & v_{T L}(t)
\end{array}\right]^{T}
$$

and the input current $u(t)=i_{T}(t)$. Here, the set of differential equations contains the expressions (1), (8), and (9).

The corresponding output equation (10) is stated as

$$
v_{T}(t)=h(\mathbf{x}(t), u(t)) .
$$

Since the battery is constantly exposed to external effects such as temperature variations and aging-which cannot be accounted for in detail in the above-mentioned model- the influence of these effects is described in a sufficiently accurate way by variations of the battery parameters. This leads to the extended battery model described in the following.

2.2. Extended battery model. As shown in Fig. 11 parameter variations are assumed to have an impact on the series resistance $R_{S}(t)$ as well as on the RC subnetwork parameters $R_{T S}(t), C_{T S}(t), R_{T L}(t)$, and $C_{T L}(t)$, as indicated by the gray boxes around the corresponding network components. This assumption helps to preserve the structure of the nominal battery model by taking into account aging effects in terms of additional parameters $\alpha(t), \beta(t)$, and $\gamma(t)$ (Rauh et al., 2010; Rauh and Aschemann, 2012).

Commonly, the series resistance $R_{S}(t)$ of the battery increases to a value $\tilde{R}_{S}(t)$ due to aging. This motivates the use of the parameter $\alpha(t)$ according to the definition

$$
\tilde{R}_{S}(t)=\alpha(t) \cdot\left(R_{S a} \cdot e^{R_{S b} \cdot \sigma(t)}+R_{S c}\right),
$$

where $\alpha=1$ denotes the nominal system behavior. Similarly, both RC subnetwork models are extended to include aging effects by the parameters $\beta(t)$ and $\gamma(t)$, expressing changes in the corresponding time constants and resistances. The new mathematical models for the RC subnetworks are then given by

$$
\tilde{v}_{T S}(t)=\beta(t) \cdot R_{T S}(t) \cdot\left(i_{T}(t)-C_{T S}(t) \cdot \dot{\tilde{v}}_{T S}(t)\right)
$$

and

$$
\tilde{v}_{T L}(t)=\gamma(t) \cdot R_{T L}(t) \cdot\left(i_{T}(t)-C_{T L}(t) \cdot \dot{\tilde{v}}_{T L}(t)\right) .
$$

As for the variable $\alpha(t)$, also the parameters $\beta(t)$ and $\gamma(t)$ are set to $\beta=1$ and $\gamma=1$ for nominal operating 
conditions, for which $\tilde{v}_{T S}(t)=v_{T S}(t)$ and $\tilde{v}_{T L}(t)=$ $v_{T L}(t)$ hold.

Since the aging parameters are characterized by time constants that are larger by several orders of magnitude than the remaining system dynamics, they can be described by independent integrator disturbance models

$$
\begin{aligned}
\dot{\alpha}(t) & =0, \\
\dot{\beta}(t) & =0, \\
\dot{\gamma}(t) & =0 .
\end{aligned}
$$

The differential equations that describe the extension of the system model (11) and (12) can be written in compact form according to

$$
\dot{\tilde{\mathbf{x}}}(t)=\tilde{\mathbf{f}}(\tilde{\mathbf{x}}(t), u(t)) .
$$

The variation rates of the extended state vector

$$
\tilde{\mathbf{x}}(t):=\left[\begin{array}{llllll}
\sigma(t) & \tilde{v}_{T S}(t) & \tilde{v}_{T L}(t) & \alpha(t) & \beta(t) & \gamma(t)
\end{array}\right]^{T}
$$

are expressed by Eqns. (1) and (15)-(19), where the new output is given by

$$
\tilde{v}_{T}(t)=v_{O C}(t)-i_{T}(t) \cdot \tilde{R}_{S}(t)-\tilde{v}_{T S}(t)-\tilde{v}_{T L}(t)
$$

as a generalization of (10). This equation is abbreviated in the following by

$$
\tilde{y}(t)=\tilde{v}_{T}(t)=\tilde{h}(\tilde{\mathbf{x}}(t), u(t)) .
$$

In the remainder of this paper, the variables available by measurements are the terminal voltage $\tilde{v}_{T}(t)$ and the battery current $i_{T}(t)$. All other states of the battery are assumed to be inaccessible in a direct way.

\section{Observer and filter design}

Luenberger-type observers and EKF techniques which are based on a continuous-time implementation of the extended battery model derived in Section 2.2 are proposed in the following. State observer techniques are utilized to estimate all components of the extended state vector $\tilde{\mathbf{x}}(t)$ in a deterministic way. To be able to estimate all components of $\tilde{\mathbf{x}}(t)$, it is necessary that the model be fully state observable (Zeitz, 1987; Krener and Isidori, 1983). Local observability can be verified by using Kalman's observability criterion, where the observability matrix is computed for the nonlinear system model after a linearization at the currently estimated state. Further approaches for the analysis of observability of nonlinear dynamic systems can be found in the work of Zhirabok and Shumsky (2012).

Using only $\tilde{v}_{T}(t)$ and $i_{T}(t)$ as available data for an observer, the extended battery model (20) with 23) is unobservable if it is linearized at a fixed operating point before the observer is designed. This difficulty can be overcome by designing an observer that directly accounts for the nonlinear system dynamics. This can be achieved by extending the system output (terminal voltage $\left.\tilde{v}_{T}(t)\right)$ by its first three low-pass filtered time derivatives (with time constants that are significantly smaller than the system dynamics) according to (Rauh et al., 2010)

$$
\begin{aligned}
\tilde{\mathbf{y}}(t) & =\tilde{\mathbf{h}}(\tilde{\mathbf{x}}(t), u(t)) \\
& =\left[\begin{array}{llll}
\tilde{v}_{T}(t) & \dot{\tilde{v}}_{T}(t) & \ddot{\tilde{v}}_{T}(t) & \ddot{\tilde{v}}_{T}(t)
\end{array}\right]^{T} .
\end{aligned}
$$

Here, the function $\tilde{\mathbf{h}}(\tilde{\mathbf{x}}(t), u(t))$ contains symbolic expressions for the time derivatives of $\tilde{v}_{T}(t)$, which are computed recursively by an evaluation of the term

$$
\tilde{v}_{T}^{(i)}(t)=\frac{\mathrm{d}^{i} \tilde{v}_{T}(t)}{\mathrm{d} t^{i}}=\left(\frac{\partial \tilde{v}_{T}^{(i-1)}(t)}{\partial \tilde{\mathbf{x}}(t)}\right)^{T} \cdot \dot{\tilde{\mathbf{x}}}(t)
$$

for $i \geq 1$, where $\dot{u}(t) \approx 0$ has been assumed.

Using techniques for symbolic formula manipulation, it can be shown that the observability mapping (Rauh et al., 2009; Isidori, 1995) contains additional information that is not present in the cases in which the system model is linearized first. Moreover, the Jacobian of this observability mapping is a matrix of full rank, corresponding to a sufficient criterion for local observability.

Therefore, the following nonlinear Luenberger-type state observer and both EKF variants considered make use of the extended output definition $\tilde{\mathbf{y}}(t)$ that is given by (24) and (25).

An illustrative example about the dynamic extension of the output equations according to (24) is given in Appendix A. This example aims at highlighting how local observability can be achieved by the corresponding procedure.

3.1. Nonlinear gain-scheduled Luenberger-type state observer. Using the extended battery model (20)-(24), the observer ansatz is given by the following set of equations (Rauh et al., 2010):

$$
\begin{aligned}
\dot{\tilde{\mathbf{x}}}(t) & =\tilde{\mathbf{f}}(\hat{\tilde{\mathbf{x}}}(t), u(t))+\mathbf{L}_{L B} \cdot(\tilde{\mathbf{y}}(t)-\hat{\tilde{\mathbf{y}}}(t)), \\
\hat{\tilde{\mathbf{y}}}(t) & =\tilde{\mathbf{h}}(\hat{\tilde{\mathbf{x}}}(t), u(t)),
\end{aligned}
$$

where $\hat{\tilde{\mathbf{x}}}(0)$ and $\tilde{\mathbf{x}}(0)$ are the initial conditions of the battery model included in the observer and the real battery system, respectively. Note that the observer design has to guarantee the convergence of $\hat{\widetilde{\mathbf{x}}}(t)$ towards $\tilde{\mathbf{x}}(t)$, even in the cases in which $\hat{\tilde{\mathbf{x}}}(0) \neq \tilde{\mathbf{x}}(0)$ holds.

In (26), a parallel model $\tilde{\mathbf{f}}(\hat{\tilde{\mathbf{x}}}(t), u(t))$ of the system dynamics is included in the differential equations of the observer which is corrected online on the basis of 
measured data by a feedback of the output error $\Delta \tilde{\mathbf{y}}(t):=$ $\tilde{\mathbf{y}}(t)-\hat{\tilde{\mathbf{y}}}(t)$.

To guarantee that the state estimation error $\Delta \tilde{\mathbf{x}}(t):=$ $\hat{\mathbf{x}}(t)-\hat{\tilde{\mathbf{x}}}(t)$ and as well as the output error $\Delta \tilde{\mathbf{y}}(t):=$ $\hat{\mathbf{y}}(t)-\hat{\tilde{\mathbf{y}}}(t)$ converge to zero, the error dynamics have to be asymptotically stable. This can be achieved by minimizing the integral cost function

$$
J=\int_{0}^{\infty}\left(\Delta \tilde{\mathbf{x}}(t)^{T} \mathbf{Q}_{L B} \Delta \tilde{\mathbf{x}}(t)+\Delta \tilde{\mathbf{y}}(t)^{T} \mathbf{R}_{L B} \Delta \tilde{\mathbf{y}}(t)\right) \mathrm{d} t
$$

with a positive semi-definite matrix $\mathbf{Q}_{L B}=\mathbf{Q}_{L B}^{T} \geq 0$ and a positive definite matrix $\mathbf{R}_{L B}=\mathbf{R}_{L B}^{T}>0$. Here, the matrices $\mathbf{Q}_{L B}$ and $\mathbf{R}_{L B}$ are chosen as constant weighting matrices. The solution of the corresponding quadratic optimization problem is given by the algebraic Riccati equation

$$
\begin{aligned}
& \mathbf{P}_{L B}\left(\sigma^{*}\right) \mathbf{C}\left(\sigma^{*}\right)^{T} \mathbf{R}_{L B}^{-1} \mathbf{C}\left(\sigma^{*}\right) \mathbf{P}_{L B}\left(\sigma^{*}\right) \\
& -\mathbf{A}\left(\sigma^{*}\right) \mathbf{P}_{L B}\left(\sigma^{*}\right)-\mathbf{P}_{L B}\left(\sigma^{*}\right) \mathbf{A}\left(\sigma^{*}\right)^{T}-\mathbf{Q}_{L B}=\mathbf{0}
\end{aligned}
$$

with a symmetric, positive definite matrix $\mathbf{P}_{L B}\left(\sigma^{*}\right)$.

Equation (29) is evaluated offline for fixed values $0 \leq \sigma^{*} \leq 1$ of the state of charge. During the solution of the algebraic Riccati equation (29), this value $\sigma^{*}$ is treated as a gain-scheduling parameter with the matrices

$$
\mathbf{A}\left(\sigma^{*}\right):=\left.\frac{\partial \tilde{\mathbf{f}}(\tilde{\mathbf{x}}(t), u(t))}{\partial \tilde{\mathbf{x}}}\right|_{\sigma=\sigma^{*}}
$$

and

$$
\mathbf{C}\left(\sigma^{*}\right):=\left.\frac{\partial \tilde{\mathbf{h}}(\tilde{\mathbf{x}}(t), u(t))}{\partial \tilde{\mathbf{x}}}\right|_{\sigma=\sigma^{*}} .
$$

The matrices (30) and (31) denote the Jacobians of the state and output equations, respectively. During their evaluation, all state variables-except for $\sigma(t)$-as well as the input current $i_{T}(t)$ are set to a fixed representative operating point of the battery.

In such a way, the offline solution of (29) and the corresponding evaluation of the observer gain matrix

$$
\mathbf{L}_{L B}\left(\sigma^{*}\right)=\mathbf{P}_{L B}\left(\sigma^{*}\right) \mathbf{C}\left(\sigma^{*}\right)^{T} \mathbf{R}_{L B}^{-1}
$$

become possible.

Note that this offline evaluation of the Riccati equation is performed under the consideration of nominal parameter values $\alpha=\beta=\gamma=1$. This simplifying assumption is removed in the following by the two presented variants of the EKF.

\footnotetext{
${ }^{1}$ An outline of this solution procedure can be found in Appendix B
}

Using the offline computed matrices $\mathbf{L}_{L B}\left(\sigma^{*}\right)$, the actual observer gain $\mathbf{L}_{L B}(\hat{\sigma})$ is adjusted online by means of a linear interpolation of the corresponding matrix entries, where the current state-of-charge estimate $\hat{\sigma}(t)$ serves as a substitute for its unknown, true value $\sigma(t)$.

The performance of this gain-scheduled Luenberger-type state observer is compared for both nominal and disturbed system parameters with an EKF that utilizes a continuous-time model of the battery. Note that the cost function (28) exploits the analogy between the linear-quadratic control and state observer design (cf. Appendix B). If the solution of the matrix Riccati equation (29) is compared with the matrix Riccati differential equation in (38), it can be moreover seen that the design criterion for the Luenberger-type observer structurally corresponds to the steady-state solution of a continuous-time extended Kalman filter design. However, Eqns. 28 and (29) do not contain any stochastic system model. Instead, the matrices $\mathbf{Q}_{L B}$ and $\mathbf{R}_{L B}$ are rather chosen in such a way as to represent the level of confidence in the measured variables and to minimize the estimation errors over reasonable time intervals.

3.2. Extended Kalman filter design. Discrete-time implementations of EKFs for the purpose of state of charge estimation have been described by Bhangu et al. (2005), Junping et al. (2009) and Plett (2004a; 2004b; 2004c). In contrast, two EKF implementations are considered in the following that utilize the continuous-time battery model directly. In the first case, a CT-EKF that contains a continuous-time model with continuous-time measurements is described. In the second case, an H-EKF is proposed. The letter employs a continuous-time system model with the practically relevant case of discrete-time measurements.

\subsubsection{Continuous-time extended Kalman filter.} For the nonlinear extended battery model described by (20)-24), the CT-EKF design is based on reformulating the state and measurement equations (20) and (24) according to the stochastic model

$$
\begin{aligned}
\mathrm{d} \tilde{\mathbf{x}}(t) & =\tilde{\mathbf{f}}(\tilde{\mathbf{x}}(t), u(t)) \mathrm{d} t+\mathbf{Q}_{K F}^{\frac{1}{2}} \mathrm{~d} \mathbf{w}_{p}(t), \\
\tilde{\mathbf{y}}(t) \mathrm{d} t & =\tilde{\mathbf{h}}(\tilde{\mathbf{x}}(t), u(t)) \mathrm{d} t+\mathbf{R}_{K F}^{\frac{1}{2}} \mathrm{~d} \mathbf{w}_{m}(t),
\end{aligned}
$$

where $\mathbf{Q}_{K F}^{\frac{1}{2}}$ and $\mathbf{R}_{K F}^{\frac{1}{2}}$ are the volatility parameters that characterize the process and measurement noise. Moreover, the noise processes $\mathrm{d} \mathbf{w}_{p}$ and $\mathrm{d} \mathbf{w}_{m}$ are then described by standard Brownian motions (Wiener processes) with zero mean. The need for including the process noise $\mathbf{w}_{p}(t)$ arises due to modeling inaccuracies and a lack of an explicit aging model for the parameters $\alpha, \beta$, and $\gamma$. Here, the standard deviation of the system noise has to be chosen such that it covers all typical model 
variations that are not explicitly accounted for in the deterministic system part $\tilde{\mathbf{f}}(\tilde{\mathbf{x}}(t), u(t))$. Additionally, the measurement noise $\mathbf{R}_{K F}^{\frac{1}{2}} \mathrm{~d} \mathbf{w}_{m}(t)$ represents inaccuracies and errors resulting from the use of low-pass filtered derivatives in (24).

As shown in the following, the design of the CT-EKF involves the linearization of the nonlinear function $\tilde{\mathbf{f}}(\tilde{\mathbf{x}}(t), u(t))$ and the output function $\tilde{\mathbf{h}}(\tilde{\mathbf{x}}(t), u(t))$ at the current state estimate $\hat{\tilde{\mathbf{x}}}(t)$. For the nonlinear system described by (33) and (34), the CT-EKF can be implemented by the following set of equations (Mohinder and Andrews, 2001; Rauh and Aschemann, 2012; Stengel, 1994):

$$
\begin{aligned}
\dot{\hat{\tilde{\mathbf{x}}}}(t)= & \tilde{\mathbf{f}}(\hat{\tilde{\mathbf{x}}}(t), u(t))+\mathbf{L}_{K F} \cdot(\tilde{\mathbf{y}}(t)-\hat{\tilde{\mathbf{y}}}(t)), \\
\mathbf{F}(t)= & \left.\frac{\partial \tilde{\mathbf{f}}(\tilde{\mathbf{x}}(t), u(t))}{\partial \tilde{\mathbf{x}}}\right|_{\tilde{\mathbf{x}}(t)=\hat{\tilde{\mathbf{x}}}(t)}, \\
\mathbf{H}(t)= & \left.\frac{\partial \tilde{\mathbf{h}}(\tilde{\mathbf{x}}(t), u(t))}{\partial \tilde{\mathbf{x}}}\right|_{\tilde{\mathbf{x}}(t)=\hat{\tilde{\mathbf{x}}}(t)}, \\
\dot{\mathbf{P}}_{K F}(t)= & \mathbf{F}(t) \mathbf{P}_{K F}(t)+\mathbf{G}(t) \mathbf{Q}_{K F}(t) \mathbf{G}^{T}(t) \\
& -\mathbf{P}_{K F}(t) \mathbf{H}^{T}(t) \mathbf{R}_{K F}^{-1}(t) \mathbf{H}(t) \mathbf{P}_{K F}(t) \\
& +\mathbf{P}_{K F}(t) \mathbf{F}^{T}(t), \\
\mathbf{G}(t)= & \mathbf{I}, \\
\mathbf{L}_{K F}(t)= & \mathbf{P}_{K F}(t) \mathbf{H}^{T}(t) \mathbf{R}_{K F}^{-1}(t),
\end{aligned}
$$

where the error covariance matrix $\mathbf{P}_{K F}(t)$ is symmetric and positive definite.

In Eqn. (35), $\hat{\tilde{\mathbf{x}}}(t)$ denotes the estimated states, $\hat{\tilde{\mathbf{y}}}(t)$ stands for the filter output, and $\mathbf{L}_{K F}(t)$ is the gain of the CT-EKF. Stability of the error dynamics has to be guaranteed as before for the initial states $\hat{\tilde{\mathbf{x}}}(0)$ and $\tilde{\mathbf{x}}(0)$, which are typically not identical, i.e., $\hat{\tilde{\mathbf{x}}}(0) \neq \tilde{\mathbf{x}}(0)$. In 36 and (37), the matrices $\mathbf{F}(t)$ and $\mathbf{H}(t)$ are the Jacobians of the state and output equations evaluated at the current state estimate $\hat{\tilde{\mathbf{x}}}(t)$, respectively.

The measurement noise covariance matrix $\mathbf{R}_{K F}(t)$, the process noise covariance matrix $\mathbf{Q}_{K F}(t)$, and the covariance $\mathbf{P}_{K F}(0)$ of the estimation error at the initial point of time represent free design parameters. The matrix $\mathbf{P}_{K F}(t)=\mathbf{P}_{K F}^{T}(t)$ is calculated by solving the matrix Riccati differential equation (38) in real time. Note that the prediction and correction steps, which are well known for discrete-time (extended) Kalman filter implementations, are coupled in the CT-EKF (Mohinder and Andrews, 2001; Stengel, 1994).

The CT-EKF is computationally more expensive than the gain-scheduled Luenberger-type state observer. An increase in the computational effort arises due to the online computation of the Kalman gain $\mathbf{L}_{K F}(t)$, which depends on the Jacobian $\mathbf{H}(t)$ as well as the solution of the matrix Riccati differential equation according to 35-39). In comparison with the Luenberger-type observer, the CT-EKF involves additional ordinary differential equations to calculate the error covariance matrix $\mathbf{P}_{K F}(t)$; see (38). However, the advantage of this extension is that the Kalman gain matrix $\mathbf{L}_{K F}(t)$ is not only scheduled as a function of the state of charge $\sigma(t)$ but also depends on the estimates for the parameters $\alpha, \beta$, and $\gamma$. This implies faster convergence to the true states and parameters in the case of large parameter uncertainties, especially for a slowly varying terminal current $i_{T}(t)$.

However, in real-life battery systems, measured data of both the terminal current and voltage are only available at fixed discrete sampling times. This is accounted for in the following subsection by the H-EKF, where the measurement model (34) is replaced by the discrete-time formulation

$$
\tilde{\mathbf{y}}\left(t_{k}\right)=\tilde{\mathbf{h}}\left(\tilde{\mathbf{x}}\left(t_{k}\right), u\left(t_{k}\right)\right)+\mathbf{w}_{m}\left(t_{k}\right),
$$

with the normally distributed discrete-time measurement noise $\mathbf{w}_{m}\left(t_{k}\right) \sim \mathcal{N}\left(0, \mathbf{R}_{h}\right)$ and the covariance $\mathbf{R}_{h}$.

3.2.2. Hybrid extended Kalman filter. To overcome the problem that the CT-EKF requires continuous values for the output voltage and the battery input current, an $\mathrm{H}$-EKF is proposed. On the one hand, this hybrid filter considers a continuous-time dynamic model (33) of the battery within the observer for the state and covariance propagation. On the other hand, discrete-time equations (40) are used in the update step when the measured data become available with a fixed sampling period. Block diagrams as well as further detailed descriptions of the methodological background of the state estimation procedures presented in this paper can be found in the work of Stengel (1994).

During the propagation step, the continuous-time model of the battery is used to estimate the states as well as the covariance matrix. The set of equations for the propagation step is given as (Stengel, 1994)

$$
\begin{aligned}
\hat{\tilde{\mathbf{x}}}^{-}\left(t_{k}\right)= & \hat{\tilde{\mathbf{x}}}^{+}\left(t_{k-1}\right)+\int_{t_{k-1}}^{t_{k}} \tilde{\mathbf{f}}\left(\hat{\tilde{\mathbf{x}}}^{-}(\tau), u(\tau)\right) \mathrm{d} \tau \\
\mathbf{P}_{h}^{-}\left(t_{k}\right)= & \mathbf{P}_{h}^{+}\left(t_{k-1}\right)+\int_{t_{k-1}}^{t_{k}}\left[\mathbf{F}_{h}(\tau) \mathbf{P}_{h}(\tau)+\mathbf{P}_{h}(\tau) \mathbf{F}^{T}(\tau)\right. \\
& \left.+\mathbf{G}(\tau) \mathbf{Q}_{K F}(\tau) \mathbf{G}^{T}(\tau)\right] \mathrm{d} \tau, \mathbf{G}(\tau)=\mathbf{I}
\end{aligned}
$$

As soon as measured data are available, the filter gain $\mathbf{L}_{h}\left(t_{k}\right)$ is computed. It is then used to update both the states $\hat{\tilde{\mathbf{x}}}^{+}\left(t_{k}\right)$ and the covariance matrix $\mathbf{P}_{h}^{+}\left(t_{k}\right)$.

According to Stengel (1994), the set of equations 
governing the update step is given by

$$
\begin{aligned}
\mathbf{L}_{h}\left(t_{k}\right)= & \mathbf{P}_{h}^{-}\left(t_{k}\right) \mathbf{H}_{h}^{T}\left(t_{k}\right)\left[\mathbf{H}_{h}\left(t_{k}\right) \mathbf{P}_{h}^{-}\left(t_{k}\right) \mathbf{H}_{h}^{T}\left(t_{k}\right)\right. \\
& \left.+\mathbf{R}_{K F}\left(t_{k}\right)\right]^{-1}, \\
\hat{\tilde{\mathbf{x}}}^{+}\left(t_{k}\right)= & \hat{\tilde{\mathbf{x}}}^{-}\left(t_{k}\right)+\mathbf{L}_{h}\left(t_{k}\right)\left[\tilde{\mathbf{y}}\left(t_{k}\right)-\hat{\tilde{\mathbf{y}}}\left(t_{k}\right)\right], \\
\mathbf{P}_{h}^{+}\left(t_{k}\right)= & {\left[\mathbf{I}_{n}-\mathbf{L}_{h}\left(t_{k}\right) \mathbf{H}_{h}\left(t_{k}\right)\right] \mathbf{P}_{h}^{-}\left(t_{k}\right) . }
\end{aligned}
$$

As for the previous filter approach, the Jacobian

$$
\mathbf{F}_{h}(\tau)=\left.\frac{\partial \tilde{\mathbf{f}}(\tilde{\mathbf{x}}(\tau), u(\tau))}{\partial \tilde{\mathbf{x}}}\right|_{\tilde{\mathbf{x}}(\tau)=\hat{\tilde{\mathbf{x}}}^{-}(\tau)}
$$

represents a linearization of the state equations, which is evaluated for the values $\hat{\tilde{\mathbf{x}}}^{-}(\tau)$ before the application of the update step. The corresponding output Jacobian

$$
\mathbf{H}_{h}\left(t_{k}\right)=\left.\frac{\partial \tilde{\mathbf{h}}\left(\tilde{\mathbf{x}}\left(t_{k}\right), u\left(t_{k}\right)\right)}{\partial \tilde{\mathbf{x}}}\right|_{\tilde{\mathbf{x}}\left(t_{k}\right)=\hat{\tilde{\mathbf{x}}}^{-}\left(t_{k}\right)}
$$

is evaluated for $\hat{\tilde{\mathbf{x}}}^{-}\left(t_{k}\right)$ at the end of the propagation step 41.

Suitable initial conditions for the state $\hat{\tilde{\mathbf{x}}}^{+}\left(t_{0}\right)$ and the error covariance matrix $\mathbf{P}_{h}^{+}\left(t_{0}\right)$ are important for a good estimation behavior. Furthermore, the noise covariance matrices $\mathbf{R}_{K F}(t)$ and $\mathbf{Q}_{K F}(t)$ represent free design parameters as in the case of the CT-EKF. In contrast to the CT-EKF, the prediction and update steps are decoupled for the hybrid filter and can, hence, be evaluated subsequently.

\section{Simulation results}

To compare the performance of the Luenberger-type state observer as well as the CT-EKF and the H-EKF, two different input current profiles are used. On the one hand, a current profile $i_{T}(t)$ with a low frequency and a small magnitude is employed. On the other hand, a rapidly changing input current with an increased magnitude is studied. Furthermore, in the case of the H-EKF, the measured data are assumed to be available in a discrete sampling interval of $10 \mathrm{~ms}$.

The observer gain matrix $\mathbf{L}_{L B}\left(\sigma^{*}\right)$ in Eqn. (32) is computed offline after discretizing the range $[0,1]$ for the state of charge $\sigma(t)$ into 20 equally spaced points. The weighting matrices $\mathbf{R}_{L B}$ and $\mathbf{Q}_{L B}$ are initialized as identity matrices. In the case of the CT-EKF and the $\mathrm{H}-\mathrm{EKF}$, large initial values of the error covariance matrix $\mathbf{P}_{K F}(0)$ are chosen to achieve a fast error convergence. It has been assumed that the correlation between the elements in the covariance matrices $\mathbf{Q}_{K F}, \mathbf{R}_{K F}, \mathbf{Q}_{h}$, and $\mathbf{R}_{h}$ is zero, hence resulting in diagonal matrices.
The covariance matrices for the process and measurement noise are set to the constant matrices

$$
\begin{aligned}
& \mathbf{Q}_{K F}=\operatorname{diag}\left(\sigma_{w 1}^{2} ; \sigma_{w 1}^{2} ; \sigma_{w 1}^{2} ; \sigma_{w 2}^{2} ; \sigma_{w 2}^{2} ; \sigma_{w 2}^{2}\right), \\
& \mathbf{R}_{K F}=\operatorname{diag}\left(0.01^{2} ; 0.01^{2} ; 0.01^{2} ; 0.01^{2}\right)
\end{aligned}
$$

for the CT-EKF and to

$$
\begin{aligned}
& \mathbf{Q}_{h}=0.01 \cdot \mathbf{Q}_{K F}, \\
& \mathbf{R}_{h}=\mathbf{R}_{K F}
\end{aligned}
$$

for the H-EKF. The values of the standard deviations $\sigma_{w 1}$ and $\sigma_{w 2}$ are chosen in such a way that the estimation quality, its convergence rate, and the robustness of the filters are sufficiently good.

To assess the performance of the Luenberger-type state observer and both the CT-EKF and the H-EKF, three different scenarios are considered for the simulation analysis. In Section 4.1, simulations are carried out for the Luenberger-type state observer as well as the CT-EKF and the H-EKF with nominal operating conditions. In Section 4.2, the performance of the observers is compared for disturbed parameters that represent aging phenomena. Since it is difficult to measure the internal resistance of a battery directly, a further simulation analysis is carried out in Section 4.3. It aims at determining the internal resistance $R_{S}(t)$ of the battery directly with the help of the proposed observers. Finally, a relative measure for the performance of the Luenberger-type observer and both the CT-EKF and the H-EKF is introduced in terms of the root of the integral square error in the state of charge estimation to provide a fair comparison between the alternative observer techniques.

4.1. Estimation of the state of charge and parameter variations under nominal operating conditions. In the first case, a nominal model of the battery is used, that is, all aging parameters $\alpha, \beta$, and $\gamma$ are initialized with 1 in both the battery and the observer model. The input current profile has a low magnitude as well as a low frequency. The extended battery model is initialized with the vector

$$
\tilde{\mathbf{x}}_{0}=\left[\begin{array}{llllll}
0.7 & 0 & 0 & 1 & 1 & 1
\end{array}\right]^{T}
$$

Moreover, the Luenberger-type state observer as well as the CT-EKF and the H-EKF are initialized with

$$
\hat{\tilde{\mathbf{x}}}_{0}=\left[\begin{array}{llllll}
0.6 & 0 & 0 & 1 & 1 & 1
\end{array}\right]^{T} .
$$

Note that $\tilde{\mathbf{x}}_{0} \neq \hat{\tilde{\mathbf{x}}}_{0}$ was chosen to visualize the capabilities of the estimation techniques to deal with initial conditions that are not exactly known. The simulation results for the Luenberger-type state observer and both the CT-EKF and the H-EKF are shown in Fig. 3 


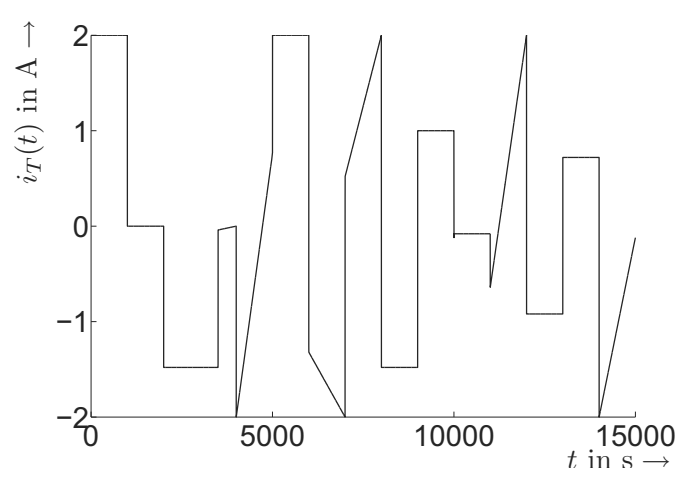

(a)

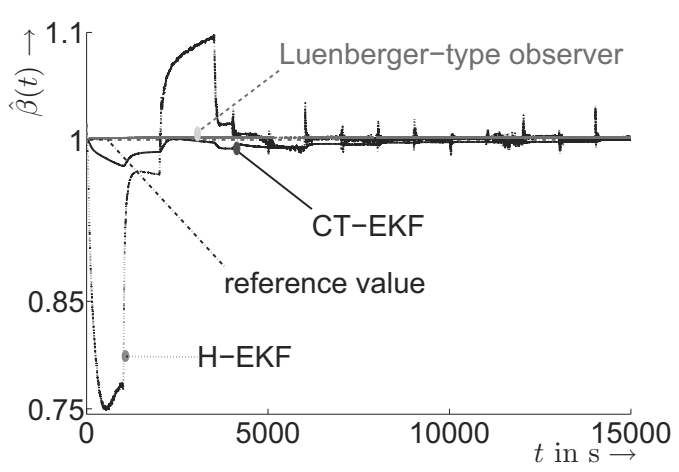

(c)

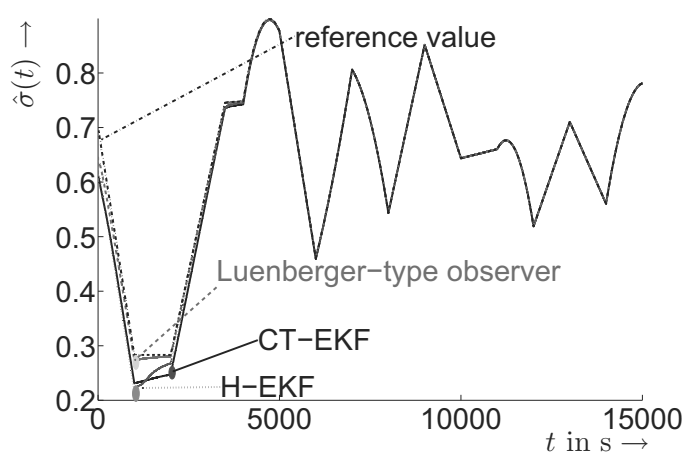

(e)

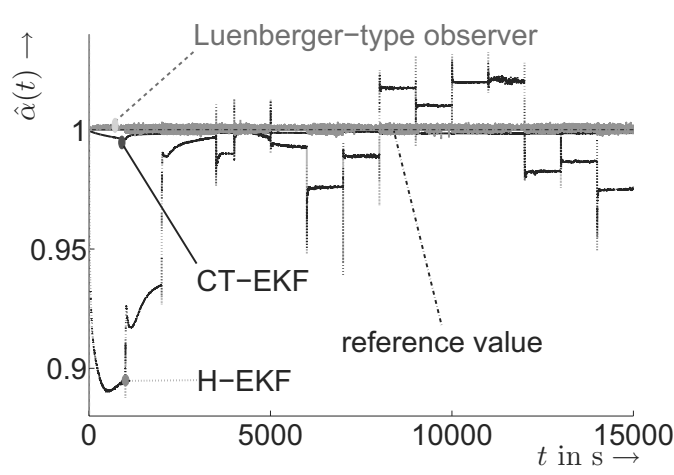

(b)

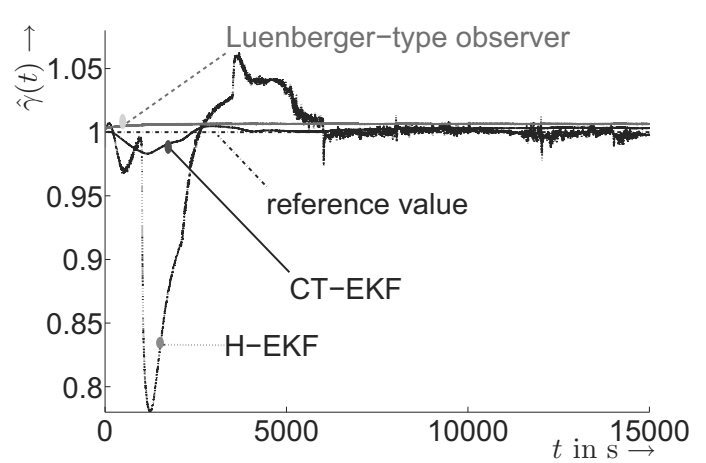

(d)

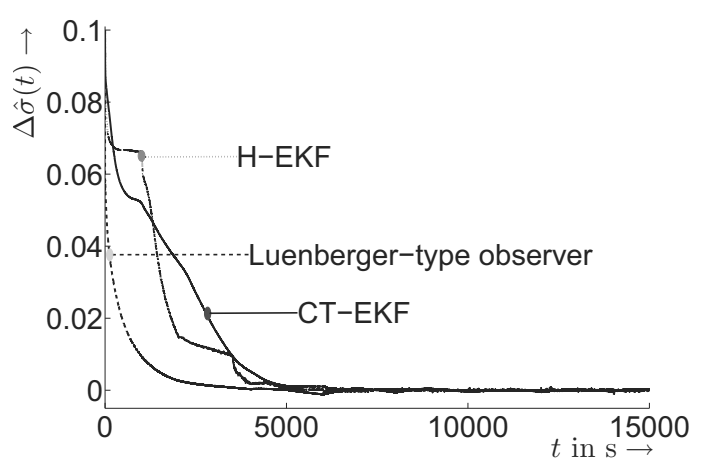

(f)

Fig. 3. Estimation results for a low frequency current with nominal parameters: terminal current $i_{T}(t)$ (a), estimate $\hat{\alpha}(t)$ for the parameter $\alpha(t)$ (b), estimate $\hat{\beta}(t)$ for the parameter $\beta(t)$ (c), estimate $\hat{\gamma}(t)$ for the parameter $\gamma(t)$ (d), estimate $\hat{\sigma}(t)$ for the state of charge $\sigma(t)$ (e), estimation error $\Delta \hat{\sigma}(t)=\sigma(t)-\hat{\sigma}(t)$ (f).

The performance of the different observers is also compared for a rapidly changing current $i_{T}(t)$. The current profile with a high frequency is given as

$$
i_{T}(t)=(-0.25+4 \cdot \operatorname{sign}(\sin (2 \pi f t)))[\mathrm{A}],
$$

where the frequency is chosen as $f=(30 \mathrm{~s})^{-1}$. The simulation results for the current profile in (52) are shown in Fig. 4

These simulation results indicate that the observed state of charge converges more quickly to the true value in the case of the Luenberger-type state observer than for the CT-EKF and the H-EKF if the current with a low frequency is used. However, for a current with a high frequency, the convergence rate is better for the CT-EKF in comparison with the Luenberger-type state observer and the H-EKF. The parameter estimates $\hat{\alpha}(t), \hat{\beta}(t)$, and $\hat{\gamma}(t)$ remain close to the assumed nominal operating conditions for all approaches in Fig. 3 . Concerning this property, the CT-EKF leads to the best behavior in Fig. 4

4.2. Estimation of the state of charge and parameter variations with aging effects. In the second case, 


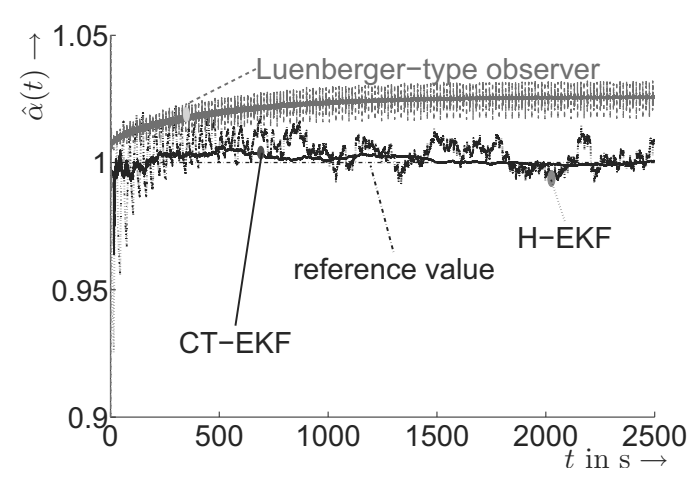

(a)

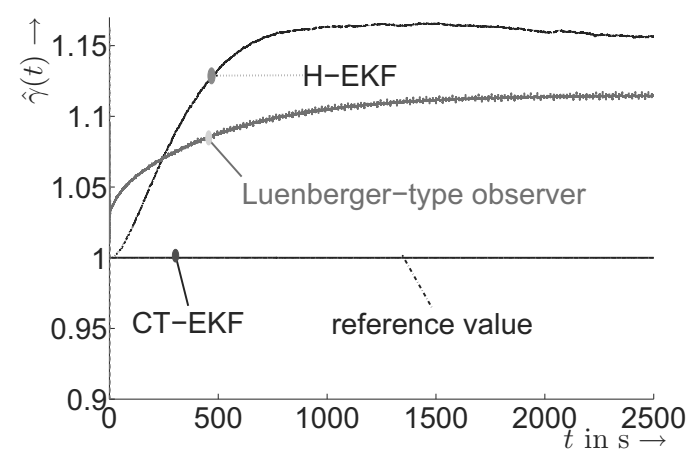

(c)

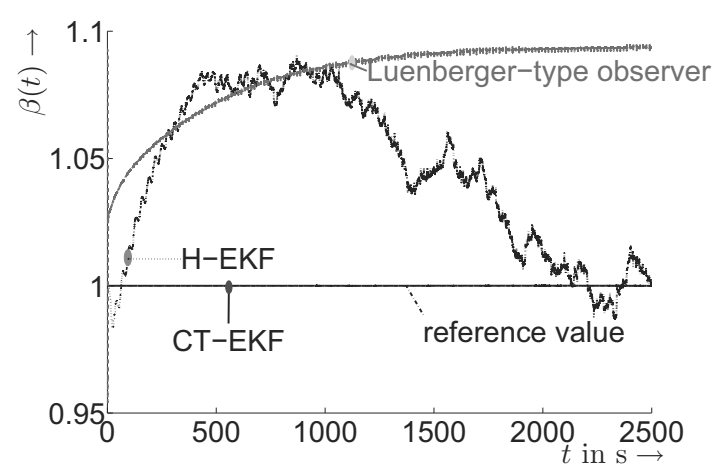

(b)

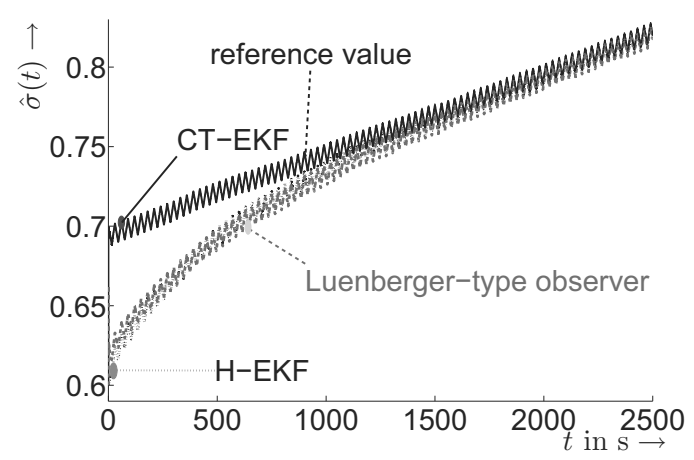

(d)

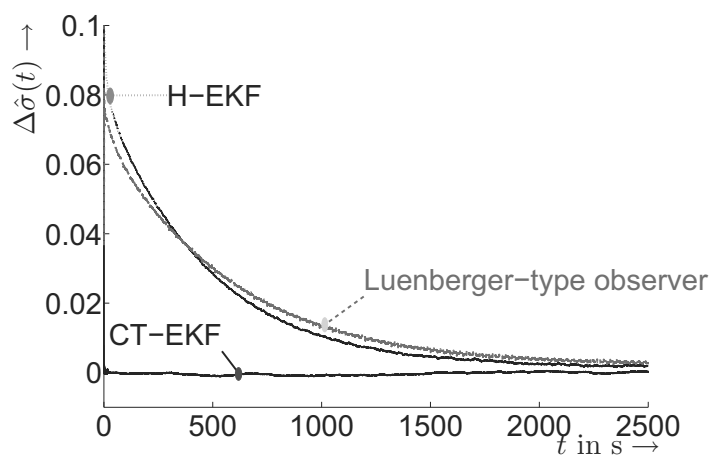

(e)

Fig. 4. Estimation results for a high frequency current with nominal parameters: estimate $\hat{\alpha}(t)$ for the parameter $\alpha(t)$ (a), estimate $\hat{\beta}(t)$ for the parameter $\beta(t)$ (b), estimate $\hat{\gamma}(t)$ for the parameter $\gamma(t)$ (c), estimate $\hat{\sigma}(t)$ for the state of charge $\sigma(t)$ (d), estimation error $\Delta \hat{\sigma}(t)=\sigma(t)-\hat{\sigma}(t)(\mathrm{e})$.

the battery parameters are subject to aging effects. Aging enlarges the internal resistance of the battery and, hence, the parameter $\alpha$ is increased. At the same time, the time constants of the RC subnetworks are also affected. Thus, the parameters $\hat{\beta}$ and $\hat{\gamma}$ are set to values differing from their nominal values $\beta$ and $\gamma$ that are used in the simulation model of the battery.

To study the effect of parameter variations, the battery is initialized with

$$
\tilde{\mathbf{x}}_{0}=\left[\begin{array}{llllll}
0.7 & 0 & 0 & 1.1 & 0.95 & 0.95
\end{array}\right]^{T}
$$

while the initial conditions for the observer and the filters are given by

$$
\hat{\tilde{\mathbf{x}}}_{0}=\left[\begin{array}{llllll}
0.6 & 0 & 0 & 1.05 & 0.85 & 0.85
\end{array}\right]^{T} .
$$

Figure 5 shows the simulation results for all estimators with the corresponding current profile with low frequency.

For the current profile with a high frequency given by (52), the simulation results are depicted in Fig. 6 According to the simulation analysis, both the CT-EKF and the H-EKF perform better in estimating the state of 


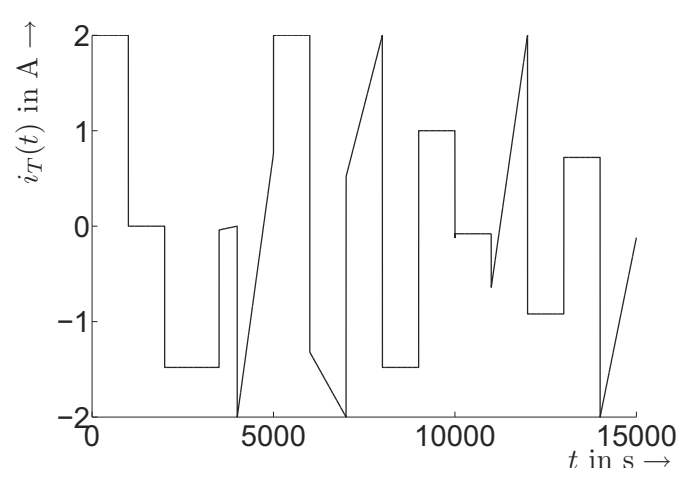

(a)

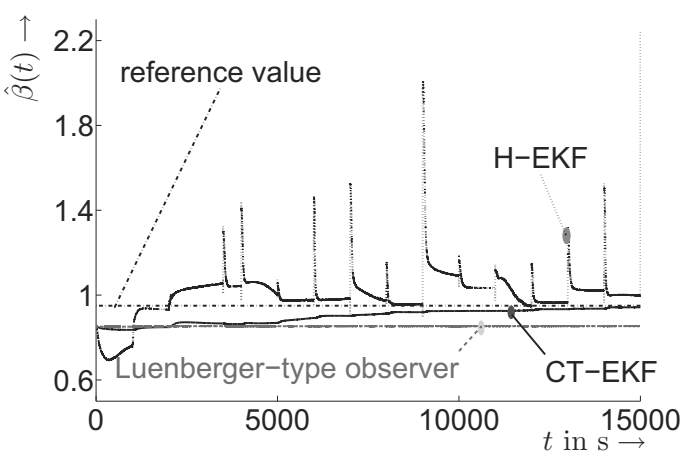

(c)

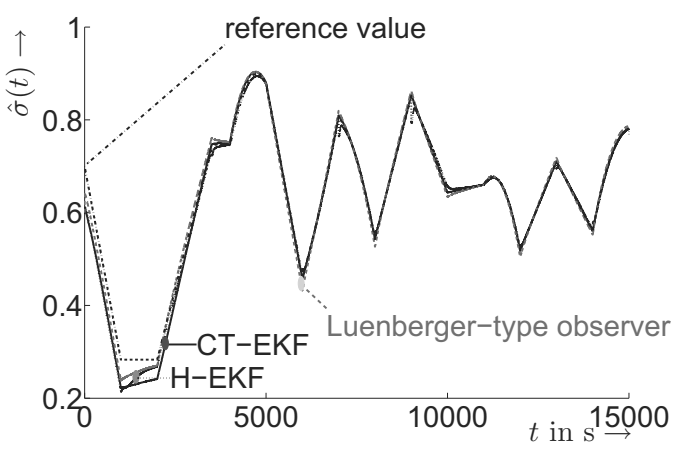

(e)

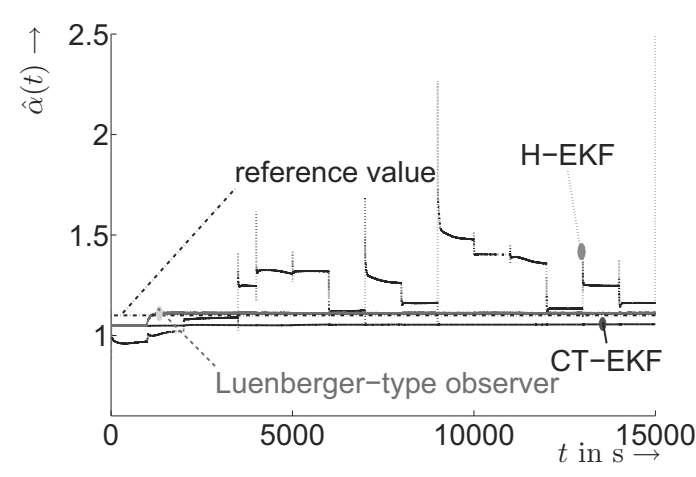

(b)

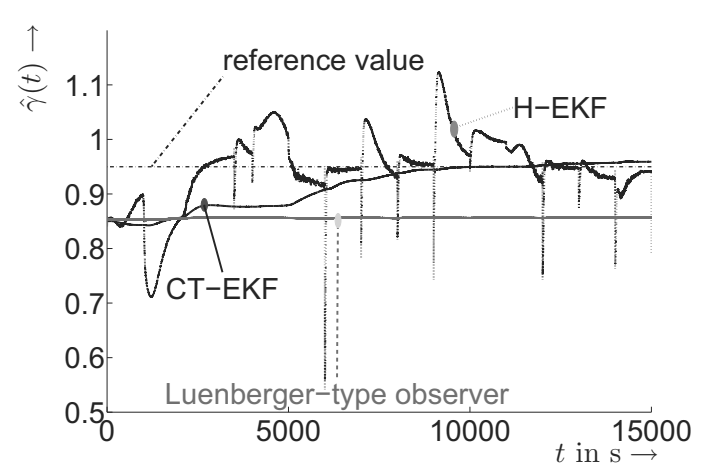

(d)

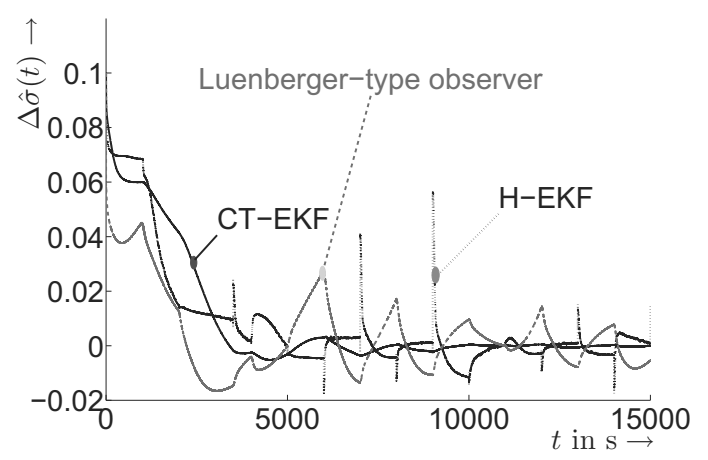

(f)

Fig. 5. Estimation results for a low frequency current with disturbed parameters: terminal current $i_{T}(t)$ (a), estimate $\hat{\alpha}(t)$ for the parameter $\alpha(t)$ (b), estimate $\hat{\beta}(t)$ for the parameter $\beta(t)$ (c), estimate $\hat{\gamma}(t)$ for the parameter $\gamma(t)$ (d), estimate $\hat{\sigma}(t)$ for the state of charge $\sigma(t)(\mathrm{e})$, estimation error $\Delta \hat{\sigma}(t)=\sigma(t)-\hat{\sigma}(t)$ (f).

charge as well as the parameter variations in comparison to the Luenberger-type observer for the low frequency current profile. However, both the CT-EKF and the H-EKF have the drawback of an increased computational effort. In contrast to the CT-EKF, the computational effort for the H-EKF is smaller because the update step is initiated only when the measured data are available. Moreover, both the CT-EKF and the H-EKF exhibit the same performance considering the estimation of the state of charge and the parameter variations. The performance improvement of the EKF with respect to the deterministic observer is due to the online computation of the observer gain $\mathbf{L}_{K F}$ and its simultaneous adaptation with respect to the parameters $\alpha, \beta$ and $\gamma$.

The rapid changes in the current profile lead to a faster state of charge convergence to the true value in the case of the CT-EKF compared with the H-EKF and the Luenberger-type observer.

4.3. Direct estimation of the internal resistance. In the third case, the internal resistance $R_{S}(t)$ of the 


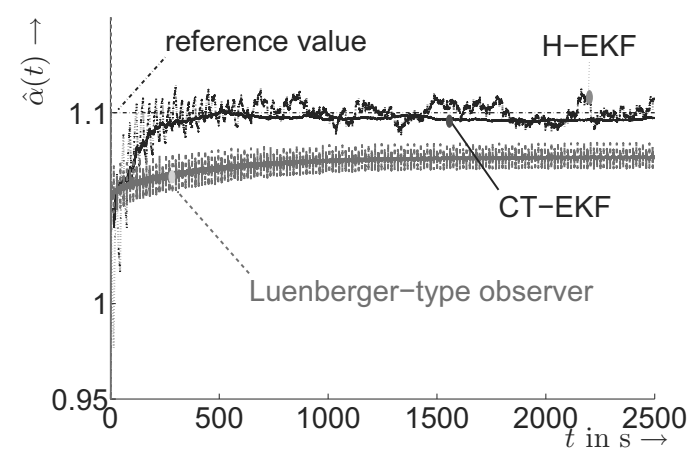

(a)

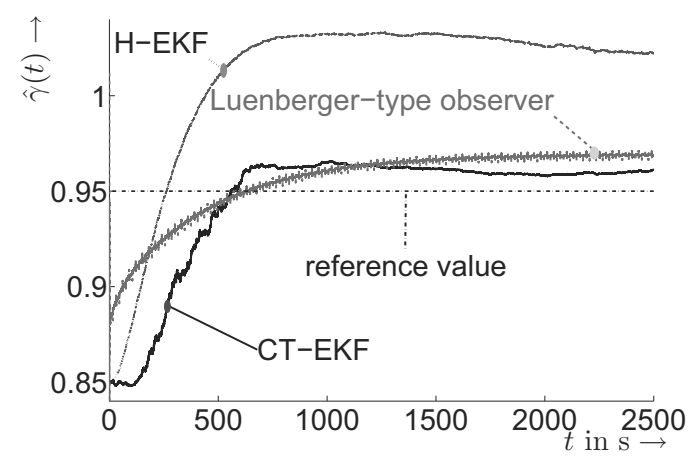

(c)

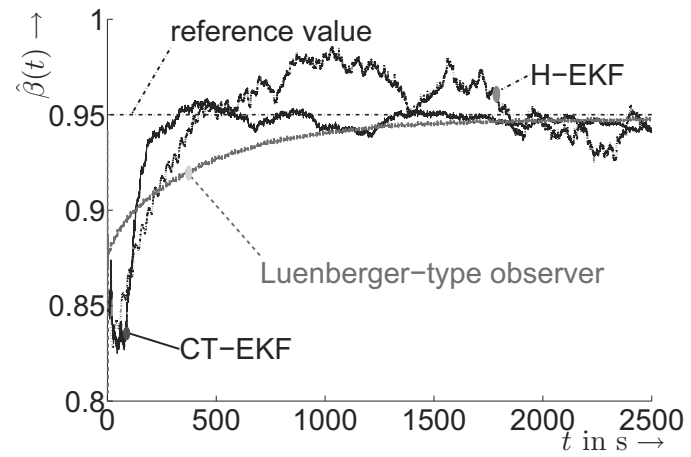

(b)

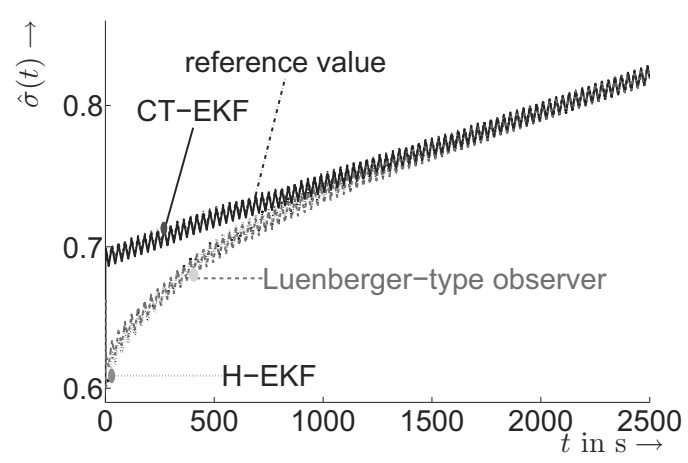

(d)

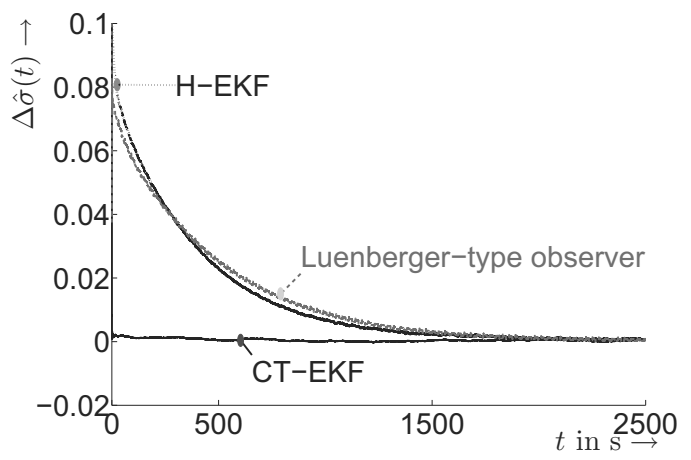

(e)

Fig. 6. Estimation results for a high frequency current with disturbed parameters: estimate $\hat{\alpha}(t)$ for the parameter $\alpha(t)$ (a), estimate $\hat{\beta}(t)$ for the parameter $\beta(t)$ (b), estimate $\hat{\gamma}(t)$ for the parameter $\gamma(t)$ (c), estimate $\hat{\sigma}(t)$ for the state of charge $\sigma(t)$ (d), estimation error $\Delta \hat{\sigma}(t)=\sigma(t)-\hat{\sigma}(t)(\mathrm{e})$.

battery is estimated directly for varying current profiles $i_{T}(t)$. The parameter $\hat{\alpha}$ is used as an estimate for $R_{S}(t)$ according to $\tilde{R}_{S}(t):=\alpha(t)$, whereas the remaining battery model given by Eqns. 20 and 22 remains unchanged. The terminal voltage for the observer is then given as

$$
\hat{\tilde{v}}_{T}(t)=v_{O C}(t)-i_{T}(t) \cdot \alpha(t)-\tilde{v}_{T S}(t)-\tilde{v}_{T L}(t) .
$$

The simulation results are shown in Figs. 7 and 8 for the current profile with a low frequency and for the rapidly changing current given by (52), respectively. In Fig. 8, the variation in the reference value of the internal resistance $R_{S}(t)$ is of small magnitude around its nominal value and, hence, it seems to be a constant line.

Again, both the CT-EKF and the H-EKF provide a superior estimation quality for the state of charge as well as the internal resistance for both current profiles, yet at the cost of an increased computational effort. In practical applications, this type of observer can replace the identification of the state of charge dependency of the 


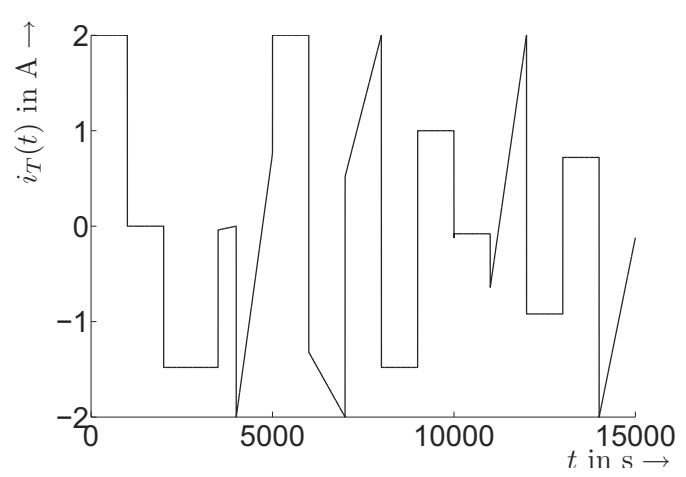

(a)

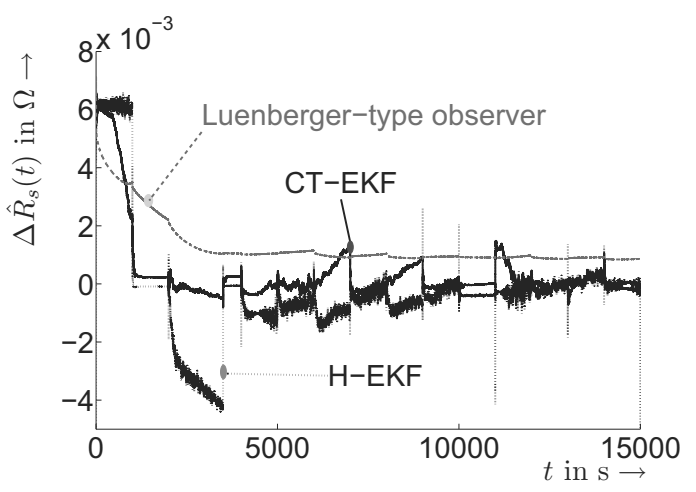

(c)

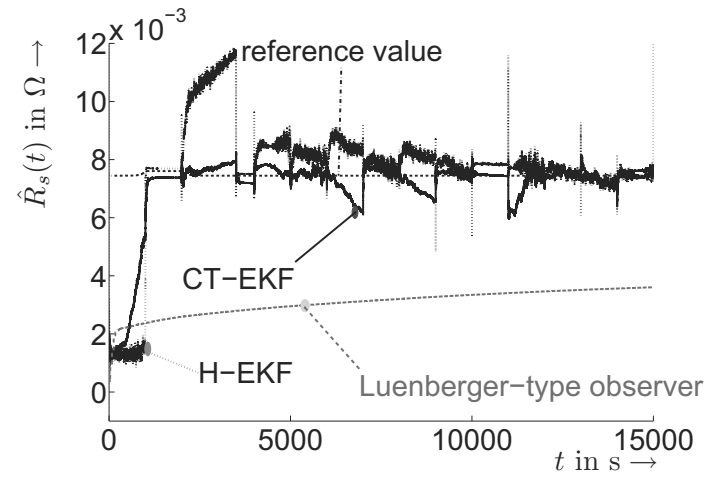

(b)

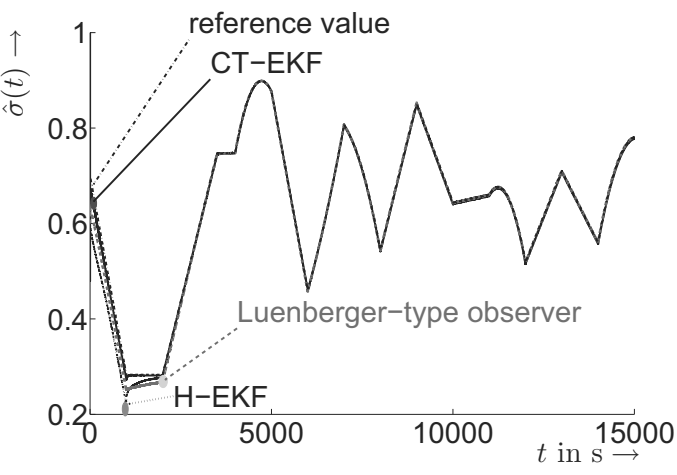

(d)

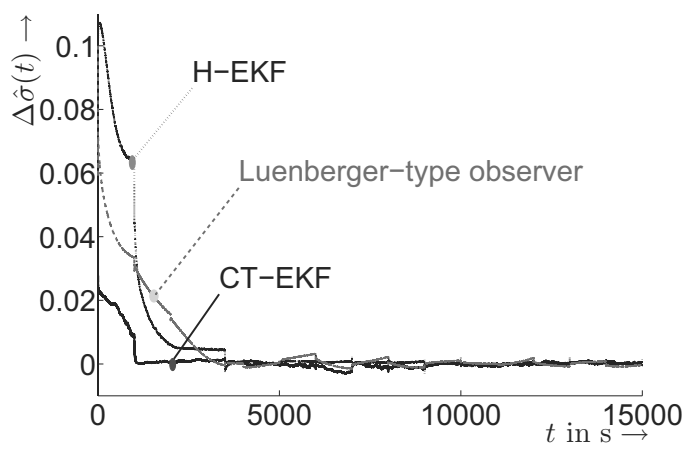

(e)

Fig. 7. Estimation results for the internal resistance $R_{S}(t)$ with a low frequency current and nominal parameters: terminal current $i_{T}(t)$ (a), estimate $\hat{R}_{S}(t)$ for the parameter $R_{S}(t)$ (b), estimation error $\Delta \hat{R}_{S}(t)=R_{S}(t)-\hat{R}_{S}(t)$ (c), estimate $\hat{\sigma}(t)$ for the state of charge $\sigma(t)(\mathrm{d})$, estimation error $\Delta \hat{\sigma}(t)=\sigma(t)-\hat{\sigma}(t)$ (e).

internal battery resistance, which is typically carried out by means of an impedance spectroscopy. Note again that the Luenberger-type observer approach is expected to be less time consuming for real-life applications than the two EKF variants.

A performance index $\chi$ (based on the root of the integral square error between the true state of charge $\sigma(t)$ and observed state of charge $\hat{\sigma}(t))$ is chosen to compare the prediction accuracy of the state of charge for the Luenberger-type observer as well as for the CT-EKF and the H-EKF for different current profiles. The performance index $\chi$ is given by

$$
\chi=\frac{1}{t_{f}} \sqrt{\int_{0}^{t_{f}}(\sigma(t)-\hat{\sigma}(t))^{2} \mathrm{~d} t},
$$

where $t_{f}$ denotes the final time for the complete length of simulation.

It can be seen from Table 1 that the performance index $\chi$ shows better results for the Luenberger-type observer with nominal operating conditions and low 


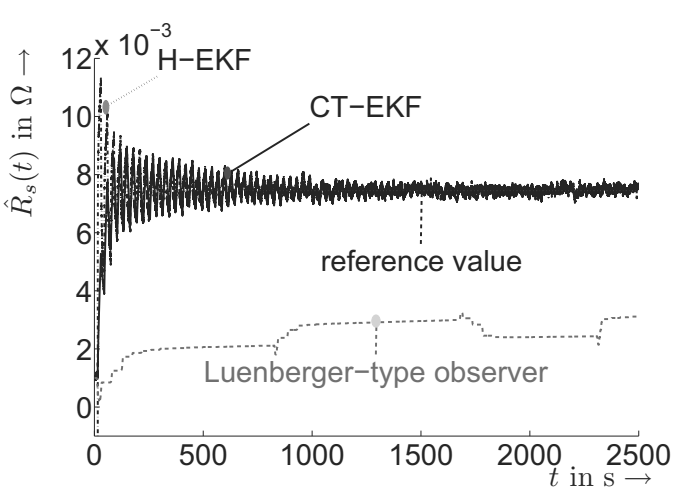

(a)

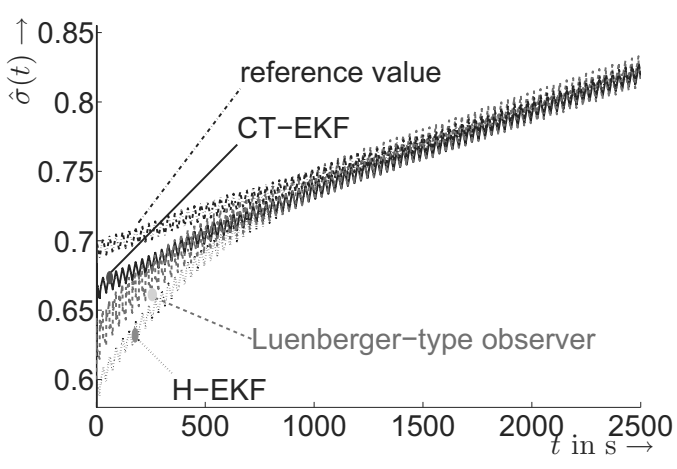

(c)

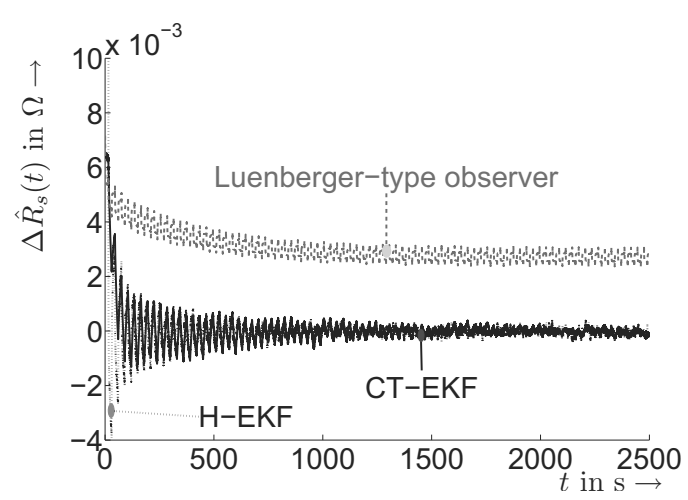

(b)

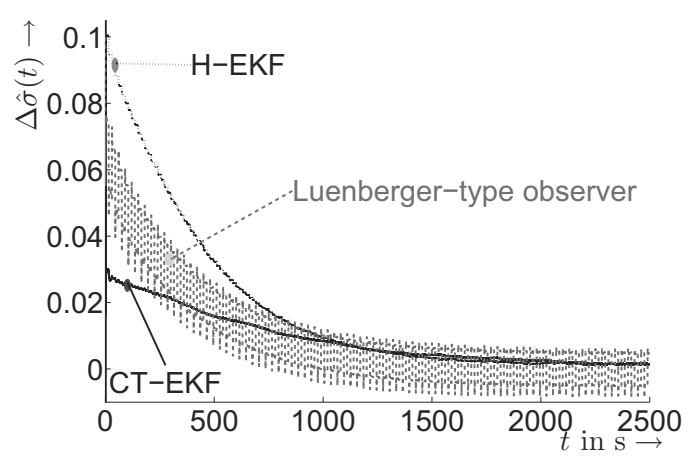

(d)

Fig. 8. Estimation results for the internal resistance $R_{S}(t)$ with a high frequency current and nominal parameters: estimate $\hat{R}_{S}(t)$ of the parameter $R_{S}(t)$ (a), estimation error $\Delta \hat{R}_{S}(t)=R_{S}(t)-\hat{R}_{S}(t)$ (b), estimate $\hat{\sigma}(t)$ of the state of charge $\sigma(t)$ (c), estimation error $\Delta \hat{\sigma}(t)=\sigma(t)-\hat{\sigma}(t)(\mathrm{d})$.

Table 1. Comparison of the performance indices $\chi$.

\begin{tabular}{|c|c|c|c|}
\hline Case study & CT-EKF $\chi$ & H-EKF $\chi$ & Luenberger-type state observer $\chi$ \\
\hline \hline Nominal parameter with a low frequency current & $9.700 \cdot 10^{-3}$ & $8.400 \cdot 10^{-3}$ & $2.000 \cdot 10^{-3}$ \\
\hline Nominal parameter with a high frequency current & $3.158 \cdot 10^{-4}$ & $1.610 \cdot 10^{-2}$ & $1.710 \cdot 10^{-2}$ \\
\hline Disturbed parameter with a low frequency current & $9.000 \cdot 10^{-3}$ & $8.500 \cdot 10^{-3}$ & $4.100 \cdot 10^{-3}$ \\
\hline Disturbed parameter with a high frequency current & $5.211 \cdot 10^{-4}$ & $1.310 \cdot 10^{-2}$ & $1.350 \cdot 10^{-2}$ \\
\hline$R_{S}(t)$ estimation with a low frequency current & $1.100 \cdot 10^{-3}$ & $7.000 \cdot 10^{-3}$ & $5.000 \cdot 10^{-3}$ \\
\hline$R_{S}(t)$ estimation with a high frequency current & $9.000 \cdot 10^{-3}$ & $1.780 \cdot 10^{-2}$ & $9.800 \cdot 10^{-3}$ \\
\hline
\end{tabular}

frequency current profiles. As high frequency profiles for the current $i_{T}(t)$ might lead to noise in the differentiation of the terminal voltage $\tilde{v}_{T}(t)$, the stochastic model used in the EKF leads to improved results.

\section{Conclusions}

In this paper, an experimentally validated control-oriented battery model has been extended by further parameter variations that cannot be determined directly on the basis of measurements to represent the battery's state of health. The state of charge, the internal resistance, and parameter variations are estimated by applying different current profiles using, first, a gain-scheduled Luenberger-type observer, second, a CT-EKF and, third, an H-EKF. Numerical simulation results show the impact of the amplitudes and variation rates of these profiles on the estimation quality.

The Luenberger-type state observer is characterized by a fast error convergence for nominal operating conditions with a slowly varying charging and discharging current profile. The computational effort required for the Luenberger-type observer is smaller than for the EKF due to the offline computation of the observer gain matrix. The 
CT-EKF and the H-EKF suppress noise resulting from the numerically differentiated terminal voltage $v_{T}(t)$ for a rapidly changing current $i_{T}(t)$ by using a stochastic model for both the system and measurement noise.

For rapidly changing currents, the CT-EKF and the H-EKF provide improved estimates for the internal battery resistance and the state of charge as well as for parameter variations due to aging. The enhanced performance of the CT-EKF and the H-EKF results from the online computation of the filter gains at the cost of its computational effort. Moreover, the H-EKF meets the basic requirements for a real-time implementation, where measurements are available only at discrete sampling times.

Future work aims at including temperature variations explicitly in the model equations to further investigate the performance of both observer and filter types. A problem that often arises when implementing the EKF is the calculation of the process noise covariance. As the process noise covariance cannot be measured directly, suitable criteria for selecting this covariance matrix will be further investigated. Furthermore, the presented observer approaches can be used for closed-loop control strategies ensuring asymptotic stability. Moreover, a sensitivity analysis with respect to the current $i_{T}(t)$ can be carried out to select the best suited current profile for a fast and robust estimation as well as a reduction in the required number of charging and discharging cycles.

\section{References}

Aylor, J., Thieme, A. and Johnso, B. (1992). A battery state-of-charge indicator for electric wheelchairs, IEEE Transactions on Industrial Electronics 39(5): 398-409.

Benger, R., Jiang, M., Beck, H., Wenzl, H., Ohms, D. and Schaedlich, G. (2009). Electrochemical and thermal modeling of lithium-ion cells for use in HEV or EV application, World Electric Vehicle Journal 3: 1-10, http: //www.evs24.org/wevajournal/ vol3/title.html

Benini, L., Castelli, G., Macii, A., Macii, E., Poncino, M. and Scarsi, R. (2001). Discrete-time battery models for system-level low-power design, IEEE Transactions on Very Large Scale Integration (VLSI) Systems 9(5): 630-640.

Bhangu, B., Bentley, P., Stone, D. and Bingham, C. (2005). Nonlinear observers for predicting state-of-charge and state-of-health of lead-acid batteries for hybrid-electric vehicles, IEEE Transactions on Vehicular Technology 54(3): 783-794.

Bo, C., Zhifeng, B. and Binggang, C. (2008). State of charge estimation based on evolutionary neural network, Journal of Energy Conversion and Management 49(10): 2788-2794.

Buller, S., Thele, M., Doncker, R. and Karden, E. (2005). Impedance based simulation models of supercapacitors and $\mathrm{Li}$-ion batteries for power electronic applications, IEEE Transactions on Industry Applications 41(3): 742-747.

Chan, C., Lo, E. and Weixiang, S. (2000). The available capacity computation model based on artificial neural network for lead-acid batteries in electric vehicles, Journal of Power Sources 87(1-2): 201-204.

Chen, M. and Rincon-Mora, G. (2006). Accurate electrical battery model capable of predicting runtime and I-V performance, IEEE Transactions on Energy Conversion 21(2): 504-511.

Chiasson, J. and Vairamohan, B. (2005). Estimating the state of charge of a battery, IEEE Transactions on Control Systems Technology 13(3): 465-470

Erdinc, O., Vural, B. and Uzunoglu, M. (2009). A dynamic lithium-ion battery model considering the effects of temperature and capacity fading, International Conference on Clean Electrical Power, Capri, Italy, pp. 383-386.

Gomadam, P., Weidner, J., Dougal, R. and White, R. (2002). Mathematical modeling of lithium-ion and nickel battery systems, Journal of Power Sources 110(2): 267-284.

Gu, W. and Wang, C. (2000). Thermal-electrochemical modeling of battery systems, Journal of The Electrochemical Society 147(8): 2910-2922.

Isidori, A. (1995). Nonlinear Control Systems, 1: An Introduction, Springer-Verlag, Berlin.

Johnson, V. (2002). Battery performance models in ADVISOR, Journal of Power Sources 110(2): 321-329.

Junping, W., Jingang, G. and Lei, D. (2009). An adaptive Kalman filtering based state of charge combined estimator for electric vehicle battery pack, Journal of Energy Conversion and Management 50(12): 3182-3186.

Kim, I. (2006). The novel state of charge estimation method for lithium battery using sliding mode observer, Journal of Power Sources 163(1): 584-590.

Klein, R., Chaturvedi, N., Christensen, J., Ahmed, J., Findeisen, R. and Kojic, A. (2012). Electrochemical model based observer design for a lithium-ion battery, IEEE Transactions on Control Systems Technology PP(99): 1-13.

Krener, J. and Isidori, A. (1983). Linearization by output injection and nonlinear observers, Journal of Systems and Control Letters 3(1): 47-52.

Leska, M., Prabel, R., Rauh, A. and Aschemann, H. (2011). Simulation and optimization of the longitudinal dynamics of parallel hybrid railway vehicles, in E. Schnieder and G. Tarnai (Eds.), FORMS/FORMAT 2010, Springer, Berlin/Heidelberg, pp. 155-164.

Levant, A. (2003). Higher-order sliding modes, differentiation and output-feedback control, International Journal of Control 76(9-10): 924-941.

Mohinder, S. and Andrews, P. (2001). Kalman Filtering: Theory and Practice-Using MATLAB, 2nd Edn., Wiley-Interscience, Hoboken, NJ.

Pang, S., Farrell, J., Du, J. and Barth, M. (2001). Battery state-of-charge estimation, American Control Conference, Arlington, VA, USA, Vol. 2, pp. 1644-1649. 
Plett, G. (2004a). Extended Kalman filtering for battery management systems of LIPB-based HEV battery packs, Part 1: Background, Journal of Power Sources 134(2): 252-261.

Plett, G. (2004b). Extended Kalman filtering for battery management systems of LIPB-based HEV battery packs, Part 2: Modeling and identification, Journal of Power Sources 134(2): 262-276.

Plett, G. (2004c). Extended Kalman filtering for battery management systems of LIPB-based HEV battery packs, Part 3: State and parameter estimation, Journal of Power Sources 134(2): 277-292.

Rauh, A. and Aschemann, H. (2012). Sensitivity-based state and parameter estimation for lithium-ion battery systems, 9th International Conference on System Identification and Control Problems, SICPRO'12, Moscow, Russia, pp. $469-485$.

Rauh, A., Minisini, J. and Hofer, E. (2009). Verification techniques for sensitivity analysis and design of controllers for nonlinear dynamic systems with uncertainties, International Journal of Applied Mathematics and Computer Science 19(3): 425-439, DOI: 10.2478/v10006-009-0035-1.

Rauh, A., Weitschat, R. and Aschemann, H. (2010). Modellgestützter Beobachterentwurf zur Betriebszustands- und Alterungserkennung für Lithium-Ionen-Batterien, VDI-Berichte 2105: Innovative Fahrzeugantriebe 2010 Die Vielfalt der Mobilitt: Vom Verbrenner bis zum E-Motor: 7. VDI-Tagung Innovative Fahrzeugantriebe, Dresden, Germany, pp. 377-382.

Remmlinger, J., Buchholz, M., Meiler, M., Bernreuter, P. and Dietmayer, K. (2011). State-of-health monitoring of lithium-ion batteries in electric vehicles by on board internal resistance estimation, Journal of Power Sources 196(12): 5357-5363.

Rong, P. and Pedram, M. (2006). An analytical model for predicting the remaining battery capacity of lithium-ion batteries, IEEE Transactions on Very Large Scale Integration (VLSI) Systems 14(5): 441-451.

Salameh, Z., Casacca, M. and Lynch, W. (1992). A mathematical model for lead-acid batteries, IEEE Transactions on Energy Conversion 7(1): 93-98.

Serrao, L., Chehab, Z., Guezennee, Y. and Rizzoni, G. (2005). An aging model of Ni-MH batteries for hybrid electric vehicles, IEEE Conference on Vehicle Power and Propulsion, Chicago, IL, USA, pp. 78-85.

Shen, Y. (2010). Adaptive online state-of-charge determination based on neuro-controller and neural network, Journal of Energy Conversion and Management 51(5): 1093-1098.

Smith, K., Rahn, C. and Wang, C. (2010). Model-based electrochemical estimation and constraint management for pulse operation of lithium ion batteries, IEEE Transactions on Control Systems Technology 3(18): 654-663.

Stengel, R. (1994). Optimal Control and Estimation, Dover Publications, Inc, Mineola, NY.
Wang, C. and Srinivasan, V. (2002). Computational battery dynamics (CBD)-electrochemical/thermal coupled modeling and multi-scale modeling, Journal of Power Sources 110(2): 364-376.

Xu, D., Jiang, B., Shi, P. (2012). Nonlinear actuator fault estimation observer: An inverse system approach via a T-S fuzzy model, International Journal of Applied Mathematics and Computer Science 22(1): 183-196, DOI: 10.2478/v10006-012-0014-9.

Zeitz, M. (1987). The extended Luenberger observer for nonlinear systems, Systems and Control Letters 9(2): 149-156.

Zhang, F., Liu, G. and Fang, L. (2008). A battery state of charge estimation method using sliding mode observer, 7th World Congress on Intelligent Control and Automation, WCICA 2008, Chongqing, China, pp. 989-994.

Zhirabok, A. and Shumsky, A. (2012). An approach to the analysis of observability and controllability in nonlinear systems via linear methods, International Journal of Applied Mathematics and Computer Science 22(3): 507-522, DOI: 10.2478/v10006-012-0038-1.

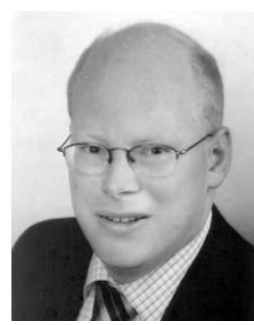

Andreas Rauh was born in Munich, Germany, in 1977. He received his diploma degree in electrical engineering and information technology from Technische Universität München, Munich, in 2001 and his Ph.D. degree (Dr.-Ing.) from the University of Ulm, Germany, in 2008. He has published more than 110 articles and chapters in edited books, international conferences and peerreviewed journals. His research interests are state and parameter estimation for stochastic and setvalued uncertainties, verified simulation of nonlinear uncertain systems, nonlinear, robust, and optimal control, interval methods for ordinary differential equations as well as differential-algebraic systems. Andreas Rauh is currently with the Chair of Mechatronics, University of Rostock, Germany, as a post-doctoral researcher. Since 2008 he has been a member of the IEEE 1788 Working Group for the Standardization of Interval Arithmetic. Moreover, in 2011, he was elected a corresponding member of the International Academy of Engineering, Moscow, Russia.

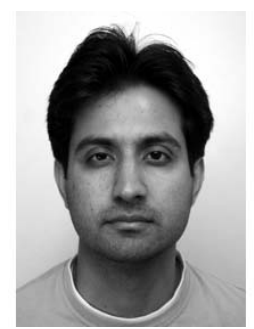

Saif Siddique Butt was born in Rawalpindi, Pakistan, in 1985. He received his B.Sc. degree in electrical engineering from UET Taxila, Pakistan, in 2007 and his M.Sc. degree in automation and robotics from Technische Universität Dortmund, Dortmund, Germany, in 2010. He is currently a Ph.D. student at the Chair of Mechatronics of the University of Rostock, Germany. His present research areas include control-oriented modeling, nonlinear control, model-predictive control and hybrid control with a focus on modeling, control and observer design for rechargeable batteries. 


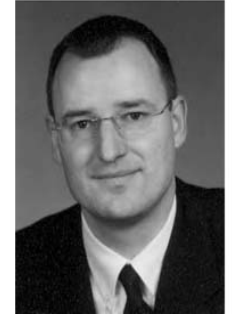

Harald Aschemann was born in Hildesheim, Germany, in 1966. He received his diploma degree in mechanical engineering from the University of Hanover, Germany, in 1994. After two years' work in research and development with a leading company in machine tools, where he focused on automated transfer systems, he joined the Department of Measurement, Control, and Microtechnology at the University of Ulm, Germany. He completed his Ph.D. (Dr.-Ing.) on optimal trajectory planning and trajectory control of an overhead travelling crane in 2001. From 2001 till 2006, he proceeded as a research associate and a lecturer at the same department. Since 2006 Harald Aschemann has been a full professor and the head of the Chair of Mechatronics at the University of Rostock, Germany. His research interests involve controloriented modelling, identification, nonlinear control, and simulation of mechatronic, robotic and thermofluidic systems. In 2011, he was elected a corresponding member of the International Academy of Engineering, Moscow, Russia.

\section{Appendix A}

\section{Observability of nonlinear systems}

Assume that a nonlinear dynamic system is described by the ordinary differential equations

$$
\begin{aligned}
& \dot{x}_{1}(t)=x_{1}(t), \\
& \dot{x}_{2}(t)=\frac{1}{2} \cdot\left(\frac{1}{x_{1}(t)}-x_{2}(t)\right)
\end{aligned}
$$

and by the output

$$
y(t)=x_{1}(t) \cdot x_{2}^{2}(t)+x_{1}(t) .
$$

Now, the goal is to check the local observability of the system model (A1), A2 at a fixed operating point $x_{1}=x_{10} \neq 0$ with $x_{2}=0$. Obviously, the linearization of the output equation (A2) at the above-mentioned operating point yields

$$
\begin{aligned}
y(t) & \left.\approx\left[\begin{array}{ll}
x_{2}^{2}+1 & 2 x_{1} x_{2}
\end{array}\right]\right|_{\substack{x_{1}=x_{10} \\
x_{2}=0}} \cdot\left[\begin{array}{l}
x_{1}(t) \\
x_{2}(t)
\end{array}\right] \\
& =\left[\begin{array}{ll}
1 & 0
\end{array}\right] \cdot\left[\begin{array}{l}
x_{1}(t) \\
x_{2}(t)
\end{array}\right]=x_{1}(t) .
\end{aligned}
$$

This linearized output equation only depends on the state variable $x_{1}(t)$. Since the first state equation in A1 is also independent of $x_{2}(t)$, the linearized system model is clearly unobservable.

Now, the system output $y(t)$ is extended by its first-order time derivative.

The computation of the derivative leads to

$$
\begin{aligned}
\dot{y}(t)= & \left(x_{2}^{2}(t)+1\right) \cdot \dot{x}_{1}(t)+2 x_{1}(t) x_{2}(t) \cdot \dot{x}_{2}(t) \\
= & \left(x_{2}^{2}(t)+1\right) \cdot x_{1}(t)+2 x_{1}(t) x_{2}(t) \\
& \cdot\left(\frac{1}{2} \cdot\left(\frac{1}{x_{1}(t)}-x_{2}(t)\right)\right)=x_{1}(t)+x_{2}(t) .
\end{aligned}
$$

Linearizing the extended system output $\mathbf{y}(t)$ at the operating point $x_{1}=x_{10} \neq 0$ with $x_{2}=0$ yields

$$
\begin{aligned}
\mathbf{y}(t) & =\left[\begin{array}{c}
y(t) \\
\dot{y}(t)
\end{array}\right]=\left[\begin{array}{c}
x_{1}(t) \cdot x_{2}^{2}(t)+x_{1}(t) \\
x_{1}(t)+x_{2}(t)
\end{array}\right] \\
& \approx\left[\begin{array}{ll}
1 & 0 \\
1 & 1
\end{array}\right] \cdot\left[\begin{array}{l}
x_{1}(t) \\
x_{2}(t)
\end{array}\right] .
\end{aligned}
$$

As can be clearly seen, this augmented output equation is locally observable for $x_{1} \neq 0$.

In analogy to this example, the state equations (24) in combination with the time derivatives of the battery's terminal voltage turn out to be a locally observable structure for which gain-scheduled state observers and Kalman filters can be designed. The $i$-th time derivatives $(i \geq 1)$ of the measured system outputs can be determined either by low-pass filtered linear approaches or by means of higher-order sliding mode derivative estimation (cf. Levant, 2003).

\section{Appendix B}

\section{Optimal state estimation}

In this appendix, the duality between the LQR control design and optimal deterministic state estimation is briefly summarized. As is well known, an optimal control design can be performed for a linear multi-input multi-output system

$$
\dot{\mathbf{x}}(t)=\mathbf{A x}(t)+\mathbf{B u}(t)
$$

by the minimization of the quadratic cost function

$$
J=\frac{1}{2} \int_{0}^{\infty}\left(\mathbf{x}^{T}(\tau) \mathbf{Q}_{C} \mathbf{x}(\tau)+\mathbf{u}^{T}(\tau) \mathbf{R}_{C} \mathbf{u}(\tau)\right) \mathrm{d} t .
$$

Solving this minimization problem (e.g., by means of Pontryagin's maximum principle or by the Hamilton-Jacobi-Bellman equation) leads to the linear state feedback controller

$$
\mathbf{u}(t)=-\mathbf{K} \mathbf{x}(t)
$$

where the controller gain $\mathbf{K}$ is determined by

$$
\mathbf{K}=\mathbf{R}_{C}^{-1} \mathbf{B}^{T} \mathbf{P}_{C} .
$$

Here, the matrix $\mathbf{P}_{C}$ is the solution of the algebraic Riccati equation

$$
\mathbf{P}_{C} \mathbf{B} \mathbf{R}_{C}^{-1} \mathbf{B}^{T} \mathbf{P}_{C}-\mathbf{A}^{T} \mathbf{P}_{C}-\mathbf{P}_{C} \mathbf{A}-\mathbf{Q}_{C}=\mathbf{0} .
$$

Hence, the closed-loop control system has the structure

$$
\dot{\mathbf{x}}(t)=(\mathbf{A}-\mathbf{B K}) \cdot \mathbf{x}(t) .
$$


To exploit the duality principle of the control and observer design, the error dynamics of a linear state observer can be stated as

$$
\Delta \dot{\mathbf{x}}(t)=(\mathbf{A}-\mathbf{H C}) \cdot \Delta \mathbf{x}(t),
$$

where $\Delta \mathbf{x}(t)=\mathbf{x}(t)-\hat{\mathbf{x}}(t)$ is the difference between the true and estimated states $\mathbf{x}(t)$ and $\hat{\mathbf{x}}(t)$, respectively, and $\mathbf{H}$ denotes the observer gain matrix. Using the transpose of Eqn. (B7], the error dynamics result in

$$
\Delta \dot{\mathbf{x}}^{T}(t)=\Delta \mathbf{x}^{T}(t) \cdot\left(\mathbf{A}^{T}-\mathbf{C}^{T} \mathbf{H}^{T}\right) .
$$

Therefore, Eqn. (B8) can be interpreted as a virtual control system with the input

$$
-\mathbf{H} \Delta \mathbf{y}(t),
$$

where $\Delta \mathbf{y}(t)=\mathbf{y}(t)-\hat{\mathbf{y}}(t)=\mathbf{C} \cdot(\mathbf{x}(t)-\hat{\mathbf{x}}(t))$ is the output error. Moreover, the gain $\mathbf{H}$ is determined by

$$
\mathbf{H}=\mathbf{P}_{L B} \mathbf{C}^{T} \mathbf{R}_{L B}^{-1},
$$

with

$$
\mathbf{P}_{L B} \mathbf{C}^{T} \mathbf{R}_{L B}^{-1} \mathbf{C} \mathbf{P}_{L B}-\mathbf{A} \mathbf{P}_{L B}-\mathbf{P}_{L B} \mathbf{A}^{T}-\mathbf{Q}_{L B}=\mathbf{0} \text { (B11) }
$$

and the equivalent cost function

$$
J_{L B}=\int_{0}^{\infty}\left(\Delta \mathbf{x}(t)^{T} \mathbf{Q}_{L B} \Delta \mathbf{x}(t)+\Delta \mathbf{y}(t)^{T} \mathbf{R}_{L B} \Delta \mathbf{y}(t)\right) \mathrm{d} t .
$$

The corresponding stochastic interpretation of B11 and (B12) leads to the steady-state solution of the Kalman filter design (Stengel, 1994).

Received: 27 August 2012

Revised: 14 March 2013

Re-revised: 28 April 2013 